\title{
Effects of the Presence of Organic Listing in Search Advertising
}

\author{
Lizhen $\mathrm{Xu}$ \\ Scheller College of Business, Georgia Institute of Technology, Atlanta, Georgia, 30308, lizhen.xu@scheller.gatech.edu \\ Jianqing Chen \\ Naveen Jindal School of Management, The University of Texas at Dallas, Richardson, Texas 75080, chenjq@utdallas.edu
}

Andrew Whinston

McCombs School of Business, The University of Texas at Austin, Austin, Texas 78712, abw@uts.cc.utexas.edu

\begin{abstract}
$\mathrm{T}_{\mathrm{er}}^{\mathrm{h}}$ his paper analyzes how the presence of organic listing as a competing information source affects advertisers' sponsored bidding and the equilibrium outcomes in search advertising. We consider a game-theoretic model in which two firms bid for sponsored advertising slots provided by a monopolistic search engine and then compete for consumers in price in the product market. Firms are asymmetrically differentiated in market preference and are given different exposure in organic listing aligned with their market appeal. We identify two aspects of a firm's sponsored bidding incentive, namely, the promotive and the preventive incentives. The presence of organic listing alters firms' sponsored bidding incentives such that the stronger firm has primarily preventive incentive, whereas the weaker has mainly promotive incentive. We show that the preventive incentive decreases and the promotive incentive increases as the difference in firms' market appeal decreases, and as a result, even the weaker firm may outbid the stronger competitor under such a co-listing setting. We further examine how the presence of organic listing affects the equilibrium outcomes by comparing it with a benchmark case in which there is only a sponsored list. We show that the differentiated exposure in the organic list gives the weaker advertiser chances to win a better sponsored position, which improves the overall information structure the search engine provides. As a result, the equilibrium social welfare, sales diversity, and consumer surplus increase. Although the presence of the free exposure from the organic list may reduce advertisers' sponsored bidding incentive per se, the overall effect benefits the search engine's growth in the long run.
\end{abstract}

Key words: organic listing; sponsored bidding; search advertising; information structure; asymmetric differentiation; price competition

History: Chris Dellarocas, Senior Editor; T. S. Raghu, Associate Editor. This paper was received May 23, 2009, and was with the authors for 17 months for 3 revisions. Published online in Articles in Advance August 24, 2012.

\section{Introduction}

Search advertising, in which the search engine provides advertising slots alongside general search results in a search engine results page (SERP), has proven itself as a successful advertising model in the recent decade. A typical SERP consists of two lists of hyperlinks: one is the organic list, which consists of nonadvertising links ranked in an order reflecting their relative importance or relevance; the other is the sponsored list, a list of advertising slots that are sold via auctions and form the revenue source of this advertising model. Search advertising generated $\$ 14.8$ billion revenue in the United States in 2011, which accounts for about half of the total Internet advertising revenue (IAB and PwC 2012). The huge industrial success has attracted increasing academic interest. Although a large volume of literature is devoted to better understanding the sponsored auctions both theoretically and empirically, the effects of organic listing, despite its being the origin and the major information source of search advertising, have been understudied in the existing literature. This paper aims to fill this gap by analyzing how the presence of organic listing as a competing information source may affect the sponsored bidding competition and the outcome performances of search advertising.

Two features deserve special attention in studying the role of organic listing. One is the unique information structure associated with a SERP; the other is the characteristics of the organic ranking mechanism.

In organizing the SERP, search engines typically place the organic list in a wide column on the left and the sponsored list in a narrow column on the right (and sometimes a highlighted area on the top as well). Such a co-listing structure forms two lists competing against each other for consumer attention. Experiments tracking eye movement of users viewing Google search result pages (Hotchkiss et al. 2005) show that the top organic links attract the most attention (e.g., the top three organic links are viewed 
by almost all experiment participants), whereas the top sponsored link attracts considerable attention but could be less significant than its organic counterparts (e.g., the first sponsored link is viewed by about half of the participants). In this sense, the organic list not only competes for consumer attention but even plays a dominating role in such competition. Note that those commercial websites interested in sponsored bidding may also appear in the organic list and could get significant attention from the organic list without paying anything, which raises questions such as why advertisers placed at top organic positions would still be willing to spend money on sponsored bidding, and whether the presence of such a competing list would affect the search engine's revenue.

Search engines' proprietary organic ranking rules, such as Google's PageRank-based ranking rule, are commonly believed to fairly reflect relevance or relative importance of different websites by utilizing the interlinking structure of websites and many other factors. Economic analysis also validates that websites' relative quality or importance is aligned with their equilibrium number of incoming links, which is consistent with the essence of the typical link analysis algorithm (e.g., PageRank algorithm) used by search engines (e.g., Katona and Sarvary 2008). As a result, among those commercial websites that are potential sponsored advertisers, the one with stronger market appeal is typically given a better organic position with higher exposure. We are thus interested in how such differentiated allocation of organic exposure affects different advertisers' bidding incentives and the sponsored bidding outcomes. Another related question is whether the organic ranking promotes diversification or not. As facilitating small advertisers and increasing diversity become the spirit of the dot-com era, major search engines recognize their role in "serving the long tail." ${ }^{1}$ Nevertheless, given that stronger firms are normally given more exposure in the organic list, we may wonder whether the typical organic ranking mechanism is serving the "tails" or making the big bigger.

This paper intends to capture the unique information structure of the co-listing organization and the essential feature of the organic ranking mechanism that aligns organic exposure with market appeal, so as to explicitly address the following research questions: How does the presence of organic listing as a competing information source affect different advertisers' sponsored bidding incentives and the bidding outcomes? How does it affect the equilibrium outcomes

\footnotetext{
${ }^{1}$ For example, Eric Schmidt, former CEO of the dominating search giant Google, once described the company's mission as "serving the long tail" (http://longtail.typepad.com/the_long_tail/2005/05/ google_longtail.html).
}

including search engine revenue, social welfare, and sales diversity?

We consider a game-theoretic model in which two firms in different organic positions bid for sponsored slots provided by a monopolistic search engine and then compete for consumers in price in the product market. Firms are asymmetrically differentiated in market preference. One firm is preferred by the majority of consumers in the market, whereas the other is preferred by a small portion of the market. The firm that is stronger in market appeal gets a top organic position. The top organic position attracts a high level of attention, whereas the first sponsored link attracts less but still reasonable attention level. We investigate the equilibrium outcome of the bidding competition, in which the value of sponsored slots is endogenously determined in the price competition. We then analyze the effects of the presence of organic listing on the equilibrium outcomes (including search engine revenue, social welfare, and sales diversity) by comparing with a benchmark case in which there is the sponsored list only (without the organic list).

In analyzing the bidding competition, we identify two aspects of a firm's incentive for acquiring a better sponsored position. One is the promotive incentive, meaning that winning a better sponsored position can directly increase a firm's overall exposure, which leads to more demand and higher profit. The other is the preventive incentive, meaning that a firm can effectively prevent a competitor from improving exposure by occupying a better sponsored position, so as to ensure its profit in the product market competition. With the presence of organic listing, the significant organic exposure given to the stronger firm diminishes the marginal benefit of increasing sponsored exposure, which results in little promotive incentive but mainly preventive incentive for the stronger firm. In contrast, the weaker firm's bidding incentive is primarily promotive. We show that as the firms become closer in market appeal, the preventive incentive decreases and the promotive incentive increases, and the latter exceeds the former above certain threshold. Therefore, we suggest that the presence of organic listing affects the sponsored bidding in that the differentiated organic exposure alters firms' sponsored bidding incentives and gives the weaker chances to win the sponsored bidding.

Compared to the benchmark case with no organic list, in which firms equally have both promotive and preventive incentives and the stronger firm always wins the sponsored bidding, the co-listing structure leads to a more balanced information structure in equilibrium, which improves the social welfare and the sales diversity. The revenue implications for the search engine are twofold. On the one hand, the presence of organic listing may hurt the search engine's 
immediate revenue (the revenue with the consumer base being fixed) because the extra free exposure in the organic list may reduce both firms' bidding incentives. On the other hand, organic listing increases the consumer surplus, which could benefit the growth of the search engine's consumer base in the long run. Therefore, we suggest that the organic listing trades off short-term profitability for long-term prosperity, which reflects farsighted wisdom.

To the best of our knowledge, this study is among the earliest to study the effects of organic listing in search advertising. It contributes to the recent literature on search advertising by explicitly modeling the organic list as a competing information source in the search advertising context and providing theoretical understanding of the effects of organic listing. This study also adds to the economic understanding of informative advertising by examining how an exogenous information source may affect the advertising competition. In the classical informative advertising view, advertising competition is essentially competition for information coverage (Bagwell 2007), in which the advertising channel is the major information source that conveys product information. In contrast, we suggest that in search advertising, a nonadvertising channel (i.e., the organic list) coexists with the advertising channel, serves as the major information source, and aligns its information structure with advertisers' intrinsic strength in market, all of which affect advertisers' advertising strategies in distinctive ways that any traditional advertising channel can hardly resemble.

The rest of the paper is organized as follows. We review the related literature in the next section. In $\S 3$, we lay out the model. In $\S 4$, we derive the equilibrium pricing and bidding outcomes. In $\$ 5$, we first set up a benchmark case with no organic list and derive the corresponding equilibrium outcomes. We then compare them with the equilibrium outcomes derived under the co-listing case as in $\S 4$, to illustrate the effects of the presence of organic listing. In $\$ 6$, we extend the main model along three directions: First, as the main results suggest that organic listing serves the "middles" better than the "tails," we explore the possibility of improving organic ranking for highly asymmetric markets. Second, we extend the model to a general information structure that allows flexible parameter values for different scenarios, and we show that the main results continue to hold. Finally, we discuss the possible extension to the case of multiple competing firms. Section 7 concludes the paper with discussion on managerial implications.

\section{Literature Review}

This study is rooted in the economics of informative advertising, in which advertisers compete for information coverage to deliver product information to consumers (e.g., Butters 1977, Grossman and Shapiro 1984, Stahl 1994, Stegeman 1991). As discussed previously, one distinction of our work is the inclusion of a nonadvertising channel as the major information source. Another difference is the exclusive nature of the advertising resource and the associated asymmetry in competition. Classical economic studies of advertising consider the advertising technology in which advertisers independently decide advertising levels, which makes the equilibrium outcome less sensitive to the difference among firms. Naturally, symmetric competition remains the theme of these works. In search advertising, however, attention levels of advertising slots drop rapidly from top downward, and only one advertiser can stay at the most prominent advertising position. This circumstance requires us to capture the small difference in firms' competitive strengths and to analyze the bidding outcomes explicitly. For this reason, we study the asymmetric competition among advertisers and consider asymmetric differentiation, as a combination of horizontal and vertical differentiation, among advertisers.

Our paper is also related to a growing literature of theoretical works on the bidding and pricing competition among advertisers and the optimal design of auction mechanism and payment scheme in search advertising (e.g., Athey and Ellison 2010; Dellarocas 2011; Edelman et al. 2007; Liu et al. 2010; Weber and Zheng 2007; Xu et al. 2011a, b; Zhang and Feng 2011). As those studies consider the sponsored list only, our study departs from them by considering the interplay between the two lists. For example, Weber and Zheng (2007) develop a model of search intermediary to study firms' bidding strategies and the search engine's optimal design. Because they focus on the optimal quality-weighting factor in ranking advertisers when considering the sponsored list only, their model does not capture the unique information structure under the co-listing setting and thus does not consider the interaction between the difference in organic exposure and the reactions in sponsored bidding, which is the focus of our study. Another distinction of our work is that, unlike most of the studies on sponsored bidding competition that treat the per-click value of a sponsored link as exogenously given, we endogenously investigate the valuation of sponsored positions in price competition, connecting the product market competition with the sponsored bidding competition.

A limited number of studies focus on the role of organic listing in search advertising from different angles. Katona and Sarvary (2010) study the bidding patterns in search advertising when considering organic listing. Yang and Ghose (2010) and Agarwal et al. (2012) are among the few that empirically investigate the potential interaction between organic and 
sponsored links. This paper complements their works by systematically examining the effects of organic listing as an additional information source on advertisers' bidding incentive for sponsored slots, search engines' revenue, consumer surplus, social welfare, and sales diversities in equilibrium. White (2009) studies the interaction between advertising and nonadvertising lists from a different angle: by incorporating more firms in the nonadvertising list, the intermediary can improve its quality to attract more users, but more firms in the lists lower the advertising firms' profits and may hurt the intermediary's revenue. Because all positions in both lists are considered the same and one firm cannot appear in both lists, there are no informative interactions between the two lists and no bidding competition among advertisers in his model, which differs from ours.

\section{The Model}

We consider two competing firms selling a certain type of product (or service) in the market. Their products are differentiated with asymmetric market preference. The firm whose product is favored by the majority of consumers or the mainstream of the market is denoted as $M$; the firm selling the product preferred by the minority of consumers or the niche market is termed as $N$. We assume that the two firms have the same production cost, which is normalized to zero.

There is a continuum of consumers with unit mass in the market. Each consumer has a unit demand of the product. Consumers differ in their tastes. The majority of the market, with a portion $1-\theta\left(0<\theta<\frac{1}{2}\right)$, prefer firm $M$ 's product to $N^{\prime}$ s, whereas the others, with a proportion $\theta$, prefer firm $N$ 's product. We sometimes call the former the $M$-type consumers and the latter the $N$-type consumers. Consumers derive utility $v$ from consuming their preferred product and derive a discounted utility $\tilde{k} v$ from the less preferred product, where $\tilde{k}$ is uniformly distributed over $[0,1]$ across all consumers. Without loss of generality, we normalize $v$ to 1 .

Consumers explore product information through a monopolistic search engine, which provides hyperlinks to firms' websites. Consumers are not aware of their preferences before the search process. On clicking the link of a firm, consumers visit the firm's website, see the product information and the price, and learn their valuation of that firm's product. A consumer's net utility is the utility from consuming the product minus the price of the product. Consumers will purchase a product only when it generates a net utility exceeding a certain reservation value, which is normalized to zero. For those consumers who visit both firms' websites, they purchase from the one giving a higher (positive) net utility.
The search engine returns a SERP in response to each search query. Each SERP contains two lists of hyperlinks-the organic list and the sponsored list. The organic links are ranked by a proprietary algorithm designed by the search engine, in an order that reflects websites' relative importance. Representing the mainstream of the product market, firm $M$ is listed at the $i_{M}$ th organic slot, which is around the very beginning of the organic list. In contrast, firm $N$ has a lower organic rank, $i_{N}$. Note that the difference in organic rank could be significant because of the existence of other noncommercial links (e.g., Wikipedia entries and news sites) appearing in between.

The sponsored slots are advertising positions, and firms can bid for a prominent position. The sponsored slots are sold via a second price auction, in which the firm with the highest bid wins the first sponsored slot and pays an amount equal to the second highest bid. ${ }^{2}$ The firm with the lower bid stays in the second sponsored slot, paying a reserve price that is normalized to zero for simplicity.

Consumers' click behavior on each SERP is modeled as follows. An individual consumer clicks the $i$ th organic link with probability $\alpha_{i}$ and clicks the $j$ th sponsored link with probability $\beta_{j}$. When a firm's link appears in both lists (e.g., in both the $i$ th organic slot and the $j$ th sponsored slot), an individual consumer clicks at least one of that firm's links with probability $1-\left(1-\alpha_{i}\right)\left(1-\beta_{j}\right)\left(1-\gamma_{i j}\right)$, where the extra term $\left(1-\gamma_{i j}\right)$ allows possible synergistic effects between organic and sponsored listing in attracting click-throughs. To highlight the fact that a top organic slot usually attracts most user attention and therefore firm $M^{\prime}$ s organic exposure rate $\alpha_{i_{M}}$ can be very close to 1 , we let $\alpha_{i_{M}}=1$. As a result, consumers visit firm $M^{\prime}$ 's website with probability 1 regardless of the sponsored bidding outcome. This simplification facilitates the tractability of the analysis; more importantly, the underlying spirit of this simplification is to highlight the role of the organic list as the major information source. We relax this assumption in $\S 6$, and main results continue to hold. To simplify the notation, we denote $\psi_{1} \equiv\left(1-\alpha_{i_{N}}\right)$. $\left(1-\beta_{1}\right)\left(1-\gamma_{i_{N} 1}\right)$ and $\psi_{2} \equiv\left(1-\alpha_{i_{N}}\right)\left(1-\beta_{2}\right)\left(1-\gamma_{i_{N}}\right)$ and let $0<\psi_{1}<\psi_{2}<1$. In other words, consumers visit firm $N^{\prime} \mathrm{s}$ website with probability $1-\psi_{1}$ if it wins the first sponsored position; otherwise, they visit its

\footnotetext{
${ }^{2}$ We rank advertisers based on their bids on the total payment, which is in fact consistent with the common practice. In practice, advertisers typically bid per-click unit prices and are ranked on the basis of their per-click bids and the expected click-throughs on their sponsored links. In our framework, both the search engine and the two advertisers rationally anticipate the expected click-throughs on the links placed at different positions. As a result, both advertisers make the per-click bidding decision in the same way as if they were submitting a total bid.
} 
Table 1 Summary of Notations

\begin{tabular}{|c|c|c|}
\hline Notation & Definition & Comments \\
\hline$\theta$ & $\begin{array}{l}\text { Among all consumers, } \theta \text { of them prefer } N \text { 's product, } 1-\theta \text { of them prefer } \\
M \text { 's product. }\end{array}$ & $0<\theta<\frac{1}{2}$ \\
\hline$\alpha_{i}, \beta_{j}, \gamma_{i j}$ & $\begin{array}{l}\text { If a firm stays in the } i \text { th organic position and the } j \text { th sponsored position, } \\
\text { consumers visit its website with probability } 1-\left(1-\alpha_{i}\right)\left(1-\beta_{j}\right)\left(1-\gamma_{i j}\right) \text {. }\end{array}$ & $\begin{aligned} & 0 \leq \alpha_{i} \leq 1 \\
& 0 \leq \beta_{j} \leq 1 \\
& 0 \leq\left(1-\alpha_{i}\right)\left(1-\beta_{j}\right)\left(1-\gamma_{i j}\right) \leq 1\end{aligned}$ \\
\hline$\psi_{1}, \psi_{2}$ & $\begin{array}{l}\text { If } N \text { wins the first sponsored position, consumers visit its website with } \\
\text { probability } 1-\psi_{1} ; \text { if } N \text { stays in the second sponsored position, } \\
\text { consumers visit its website with probability } 1-\psi_{2} \text {. }\end{array}$ & $\begin{aligned} & 0<\psi_{1}<\psi_{2}<1 \\
& \psi_{1}=\left(1-\alpha_{i_{N}}\right)\left(1-\beta_{1}\right)\left(1-\gamma_{i_{N} 1}\right) \\
& \psi_{2}=\left(1-\alpha_{i_{N}}\right)\left(1-\beta_{2}\right)\left(1-\gamma_{i_{N}}\right)\end{aligned}$ \\
\hline$\psi_{1}^{\prime}, \psi_{2}^{\prime}$ & $\begin{array}{l}\text { If } M \text { wins the first sponsored position, consumers visit its website with } \\
\text { probability } 1-\psi_{1}^{\prime} \text {; if } M \text { stays in the second sponsored position, } \\
\text { consumers visit its website with probability } 1-\psi_{2}^{\prime} \text {. }\end{array}$ & $\begin{array}{l}0 \leq \psi_{1}^{\prime} \leq \psi_{2}^{\prime}<1 \\
\psi_{1}^{\prime}=\left(1-\alpha_{i_{M}}\right)\left(1-\beta_{1}\right)\left(1-\gamma_{i_{M} 1}\right) \\
\psi_{2}^{\prime}=\left(1-\alpha_{i_{M}}\right)\left(1-\beta_{2}\right)\left(1-\gamma_{i_{M}{ }^{2}}\right) \\
\text { In the main model, } \psi_{1}^{\prime}=\psi_{2}^{\prime}=0\end{array}$ \\
\hline$\tilde{k}$ & Consumers' discounted utility for the less-preferred product. & $\begin{array}{l}\tilde{k} \text { is uniformly distributed over }[0,1] \\
\text { across all consumers }\end{array}$ \\
\hline$\lambda$ & $\begin{array}{l}\text { In mixed organic ranking, with probability } \lambda, N \text { stays in a prominent organic } \\
\text { position, and } M \text { stays in a less prominent organic position. }\end{array}$ & $\begin{array}{l}\qquad 0<\lambda<1 \\
\text { Pure organic ranking is a special case } \\
\text { with } \lambda=0 \text {. }\end{array}$ \\
\hline$\pi_{M}, \pi_{N}$ & Firms' profits from product sale. & \\
\hline$\Delta \pi_{M}, \Delta \pi_{N}$ & $\begin{array}{l}\text { Difference in firms' sales profit between winning the first sponsored position } \\
\text { and staying in the second sponsored position. }\end{array}$ & \\
\hline$b_{M}, b_{N}$ & Firms' bids for the first sponsored position. & Refers to the benchmark case \\
\hline & The social welfare in equilibrium. & $\sim$ Refers to the mixed organic ranking \\
\hline G & The Gini coefficient for product sales in equilibrium. & \\
\hline$I R$ & The search engine's immediate revenue in equilibrium. & \\
\hline CS & The consumer surplus in equilibrium. & \\
\hline
\end{tabular}

website with probability $1-\psi_{2}$. In this sense, $\psi_{1}$ and $\psi_{2}$ are measures of the information incompleteness within the market.

The timing of the game is as follows: In the first stage, firms submit bids for the sponsored slots. In the second stage, after observing the bidding outcome, both firms set their price simultaneously. Finally, consumers browse the SERP, sample firms' websites, and make purchase decisions. Table 1 summarizes the notations used in the paper.

\section{Equilibrium Analysis}

Along the line of backward induction, we start with the second stage price competition. We first formulate firms' market shares under a complete information setting, where all consumers are aware of the two products and know the product details and price information:

$$
\begin{aligned}
& S_{M}\left(p_{M}, p_{N}\right)=(1-\theta)\left(1-\left[p_{M}-p_{N}\right]^{+}\right)+\theta\left[p_{N}-p_{M}\right]^{+} \\
& S_{N}\left(p_{N}, p_{M}\right)=\theta\left(1-\left[p_{N}-p_{M}\right]^{+}\right)+(1-\theta)\left[p_{M}-p_{N}\right]^{+},
\end{aligned}
$$

where firms' prices $p_{M}, p_{N} \in[0,1]$, and $[\cdot]^{+}$represents $\max \{\cdot, 0\}$. An $M$-type consumer will buy product $M$ as long as $1-p_{M} \geq \tilde{k}-p_{N}$, which explains the first term of $S_{M}\left(p_{M}, p_{N}\right)$. Similarly, an $N$-type consumer will buy product $M$ only if $\tilde{k}-p_{M}>1-p_{N}$, which explains the second term of $S_{M}\left(p_{M}, p_{N}\right)$. We can interpret $S_{N}\left(p_{N}, p_{M}\right)$ in a similar way. Notice that consumers discount their less-preferred product differently, and some consumers do not have strong preference (i.e., with $\tilde{k}$ close to 1 ). Therefore, either firm can compete for these consumers with a competitive price as long as it gets exposed to them.

Similarly, we can define firms' market shares in the case of informational monopoly, where consumers are aware of only one firm's product information and price:

$$
\begin{aligned}
& A_{M}\left(p_{M}\right)=(1-\theta)+\theta\left(1-p_{M}\right) \\
& A_{N}\left(p_{N}\right)=\theta+(1-\theta)\left(1-p_{N}\right),
\end{aligned}
$$

for $p_{M}, p_{N} \in[0,1]$. In the case of $A_{M}$, for example, all $M$-type consumers buy from firm $M$, whereas $N$-type consumers buy from firm $M$ only if $\tilde{k}-p_{M} \geq 0$.

Recall that consumers visit firm $M$ 's website with probability 1 and visit firm N's website with probability $1-\psi$, where

$$
\psi= \begin{cases}\psi_{1} & \text { when } N \text { wins the first sponsored slot; } \\ \psi_{2} & \text { otherwise. }\end{cases}
$$

Note that $\psi$ reflects the informational advantage of firm $M$ over firm $N$, and winning the top sponsored slot can help firm $N$ increase its exposure by reducing $\psi$ from $\psi_{2}$ to $\psi_{1}$.

Given the information structure determined by the first stage bidding outcome (which is characterized 
by $\psi$ ), we can formulate firms' demand functions. A portion $\psi$ of consumers is aware of $M^{\prime}$ 's product only, and the other portion is aware of both products. Therefore,

$$
\begin{aligned}
& D_{M}\left(p_{M}, p_{N}\right)=\psi A_{M}\left(p_{M}\right)+(1-\psi) S_{M}\left(p_{M}, p_{N}\right) \\
& D_{N}\left(p_{N}, p_{M}\right)=(1-\psi) S_{N}\left(p_{N}, p_{M}\right) .
\end{aligned}
$$

Firms' profits can thus be written as

$$
\pi_{i}\left(p_{i}, p_{\bar{i}}\right)=p_{i} D_{i}\left(p_{i}, p_{\bar{i}}\right), \quad\{i, \bar{i}\}=\{M, N\} .
$$

Based on the best responses derived from maximizing the profit functions, we can derive the equilibrium prices in the second stage:

$$
\left\{\begin{array}{l}
p_{M}^{*}=\min \left\{\frac{2-\theta(1-\psi)}{3(1-\theta)(1-\psi)+4 \theta \psi}, 1\right\} \\
p_{N}^{*}=\frac{\theta+(1-\theta) p_{M}^{*}}{2(1-\theta)} .
\end{array}\right.
$$

Note that in equilibrium, $p_{N}^{*}<p_{M}^{*}$; that is, firm $N$, which is at a disadvantage in terms of market preference, tends to cut its price to compete for market share against its stronger competitor. On the other hand, firm $M$ tends to stay away from the intense price competition when it has significant informational advantage. In fact, $p_{M}^{*}=1$ when $\psi \geq \frac{1}{3}$, according to Equation (5). As long as its informational advantage is reasonably large, firm $M$ forgoes the price competition with $N$ and simply charges the monopoly price to fully exploit its guaranteed demand.

Next, we derive firms' equilibrium profits from the second stage price competition, $\pi_{i}^{*}(\theta, \psi)$, as a function of the market asymmetry level $\theta$ and the information incompleteness level $\psi$. By substituting $\left\{p_{M}^{*}, p_{N}^{*}\right\}$ into Equation (4), we summarize $\pi_{i}^{*}(\theta, \psi)$ in Table 2 .

A close examination of the equilibrium profit functions $\pi_{i}^{*}(\theta, \psi)$ reveals some interesting aspects of firms' bidding incentives. First, $\partial \pi_{M}^{*}(\theta, \psi) / \partial \psi>0$, which means that firm $M^{\prime}$ s equilibrium profit increases when $\psi$ increases, that is, when its competitor's exposure decreases. Therefore, although firm $M^{\prime}$ 's own exposure stays the same regardless of the sponsored bidding outcome, it is still desirable for $M$ to win the top sponsored position because it effectively prevents $N$ from increasing its exposure and

Table 2 Equilibrium Profit Functions in the Second Stage Price Competition

\begin{tabular}{ccc}
\hline & $0<\psi<\frac{1}{3}$ & $\frac{1}{3} \leq \psi<1$ \\
$\pi_{M}^{*}(\theta, \psi)$ & $\frac{[2-\theta(1-\psi)]^{2}[(1-\theta)-(1-2 \theta) \psi]}{[3(1-\theta)(1-\psi)+4 \theta \psi]^{2}}$ & $\left(\frac{1}{2}-\theta\right) \psi+\frac{1}{2}$ \\
$\pi_{N}^{*}(\theta, \psi)$ & $\frac{[(1+\theta)(1-\theta)+\theta(3 \theta-1) \psi]^{2}(1-\psi)}{(1-\theta)[3(1-\theta)(1-\psi)+4 \theta \psi]^{2}}$ & $\frac{1-\psi}{4(1-\theta)}$ \\
\hline
\end{tabular}

hence increases $M^{\prime}$ s profit. Second, $\partial \pi_{N}^{*}(\theta, \psi) / \partial \psi<0$ in most cases, which indicates that firm $N^{\prime}$ s equilibrium profit generally increases when $\psi$ decreases, that is, when N's own exposure improves. Therefore, winning a better sponsored position is profitable for firm $N$ because it improves its overall exposure level, even though it cannot affect its competitor's exposure level. Thus, we identify two different incentives for firms to bid for a better sponsored position: one is preventive incentive, the incentive to prevent competitors from improving their exposure; the other is promotive incentive, the incentive to promote its own exposure.

Note that $\partial \pi_{M}^{*}(\theta, \psi) / \partial \psi$ can thus be interpreted as the marginal preventive incentive of firm $M$, and $-\partial \pi_{N}^{*}(\theta, \psi) / \partial \psi$ reflects firm $N^{\prime}$ s marginal promotive incentive. We next examine how these incentives change with the market structure.

LEMMA 1. Both firms' equilibrium profit functions are submodular, that is, $\partial^{2} \pi_{i}^{*}(\theta, \psi) / \partial \theta \partial \psi<0, i \in\{M, N\}$.

Proof. All proofs are detailed in the appendix.

As is implied by Lemma 1 , as $\theta$ increases, $M^{\prime}$ s marginal preventive incentive $\left(\partial \pi_{M}^{*}(\theta, \psi) / \partial \psi\right)$ decreases, and $N^{\prime}$ s marginal promotive incentive $\left(-\partial \pi_{N}^{*}(\theta, \psi) / \partial \psi\right)$ increases. In other words, as the two firms get closer in market appeal, $N^{\prime}$ s benefit from increasing its own exposure increases, whereas $M^{\prime}$ 's benefit from blocking its competitor decreases. This is because when the $N$-type consumer base increases, firm $N$ becomes increasingly competitive, and it can capture a significant amount of demand once it gets exposed to the consumers. On the contrary, the market share that firm $M$ can capture reduces, even under informational monopoly. Therefore, firm $N$ becomes increasingly aggressive to win the sponsored bidding, whereas $M$ is less incentivized to maintain its informational advantage. This explains why the two firms' bidding incentives evolve in the opposite directions as market structure changes.

Based on the understanding of firms' bidding incentives, we next derive the bidding outcome in the first stage. In second-price auctions, it is well documented that bidding the true value is a weakly dominant strategy for all bidders. Therefore, in the first stage, the unique perfect equilibrium is that both firms bid their true value $b_{i}^{*}$ :

$$
\left\{\begin{array}{l}
b_{M}^{*}=\left[\pi_{M}^{*}\left(\theta, \psi_{2}\right)-\pi_{M}^{*}\left(\theta, \psi_{1}\right)\right]^{+} \\
b_{N}^{*}=\left[\pi_{N}^{*}\left(\theta, \psi_{1}\right)-\pi_{N}^{*}\left(\theta, \psi_{2}\right)\right]^{+},
\end{array}\right.
$$

which equals their respective equilibrium profit difference between winning the first sponsored slot and otherwise, bounded below at zero. Applying the results from Lemma 1, we uncover the bidding outcome as follows.

Proposition 1. In equilibrium, there exists a cutoff $\theta^{*}\left(\psi_{1}, \psi_{2}\right)$, such that when $0<\theta<\theta^{*}\left(\psi_{1}, \psi_{2}\right)$, $M$ bids 
higher and wins the first sponsored slot, and when $\theta^{*}\left(\psi_{1}, \psi_{2}\right)<\theta<\frac{1}{2}, N$ outbids its rival. Here, $\theta^{*}\left(\psi_{1}, \psi_{2}\right) \in$ $\left(0, \frac{1}{2}\right)$ and is determined by

$$
\pi_{M}^{*}\left(\theta^{*}, \psi_{2}\right)-\pi_{M}^{*}\left(\theta^{*}, \psi_{1}\right)=\pi_{N}^{*}\left(\theta^{*}, \psi_{1}\right)-\pi_{N}^{*}\left(\theta^{*}, \psi_{2}\right) .
$$

Proposition 1 shows that because the two firms' bidding incentives change with the market structure $(\theta)$ in the opposite directions, there exists a threshold $\theta^{*}$, a certain cutoff level of market asymmetry, which partitions the bidding outcome space. When $\theta$ is above that threshold, that is, when market is relatively symmetric and firms are comparable to each other, firm $N$ 's promotive incentive exceeds $M^{\prime}$ s preventive incentive, so $N$ outbids $M$ and wins the first sponsored position. When $\theta$ is below that threshold, $M^{\prime}$ 's preventive incentive dominates, and the stronger firm wins the sponsored bidding.

The analysis thus far provides understanding on how organic listing affects sponsored bidding. The organic list gives advertisers different levels of free exposure aligned with their market appeal, which leads to different types of incentive in pursuing extra sponsored exposure for different advertisers. Because these different incentives change with the market structure in different directions, the sponsored bidding outcome can be one way or another. As a result, organic listing balances the equilibrium bidding outcome such that even a weaker firm may be able to outbid its stronger rival.

It is worth pointing out that the effects of organic listing on sponsored bidding extend beyond the current model setting. Recall that we assume $\alpha_{i_{M}}=1$, which makes firm $M$ have the preventive incentive only and firm $N$ have the promotive incentive only. When this assumption is relaxed, as we discuss in $\S 6.2$, each firm has both promotive and preventive incentives for winning the sponsored bidding. In that case, the effect of the organic list is to affect the relative strength of the two incentives for different firms. For a firm at a top organic position with significant exposure, winning the sponsored bidding only marginally improves its overall exposure but prevents the competitor in a lower organic position from substantially increasing exposure. Therefore, its preventive incentive is stronger than its promotive incentive. On the contrary, by similar reasoning, for a firm at a lower organic position, its promotive incentive outweighs its preventive incentive. In this sense, the current model setting highlights the essence of interest by focusing on the extreme case, which allows us to disentangle the two otherwise intertwined incentives. As we will show, under the general information structure, the bidding outcome follows a similar pattern, and the key driver remains the same: it is the differentiated exposure given in the organic list that adjusts firms' bidding incentives and leads to a balanced bidding outcome.

Proposition 1 also provides rationale for firms in different types of markets to determine the value of a prominent sponsored slot in bidding competition. In a highly asymmetric market, the market leader should bid aggressively to win the top sponsored slot because occupying a prominent position in both the organic and sponsored list enlarges its informational dominance, ensures its advantageous position in price competition, and greatly improves its sales profit. However, when the market preference is relatively diversified, the firm that is weaker in market appeal should bid to win a prominent sponsored slot, especially when it is placed at a lower organic position with an unsatisfactory attention level. This is because its marginal benefit from improving its exposure is high in this case.

Given that firm N's bidding incentive decreases as $\theta$ goes down, it is thus worth noting that in a certain parameter region, such an incentive can fall so low that firm $N$ is not willing to bid any positive amount.

Corollary 1. Firm $N$ may have no incentive for sponsored bidding; in particular, when $\psi_{2}<\frac{1}{3}$ and $\theta<$ $(17-\sqrt{145}) / 24, b_{N}^{*}=0$.

When the market preference is highly asymmetric (i.e., $\theta$ is small), if firm $N$ can gain a reasonable level of attention from the organic link and the second sponsored link (i.e., $\psi_{2}<\frac{1}{3}$ ), it has no intention to bid for the top sponsored link at all. The reason is that, should $N$ win the first sponsored slot and increase its exposure, it would trigger an intense price war with firm $M$, which would eventually result in an even lower equilibrium profit level for firm $N$. The driving force of $N$ 's lack of bidding incentive is the unshakable advantage in organic exposure that the strong firm has.

\section{Effects of Organic Listing}

In this section, we investigate how the presence of organic listing affects the equilibrium outcomes. We first construct a case that is absent of organic listing as a benchmark and then compare the equilibrium outcomes under the benchmark case with those derived from $\S 4$ in three aspects: social welfare, sales diversity, and search engine revenue.

\subsection{A Benchmark Case}

As a benchmark, we consider a hypothetical case in which each SERP contains the sponsored list only. The benchmark case can be imagined as the search engine's choosing to display only one list of links, all of which are potential advertising slots to be sold. Similar practices can be found, for example, in early 
versions of search advertising, such as those used by Goto.com and, later, Overture.com. In contrast, we call the original case with both organic list and sponsored list the co-listing case.

To be consistent with the original model, we consider two sponsored links on a SERP. An individual consumer clicks the first sponsored link with probability $q_{1}$ and the second with probability $q_{2}\left(q_{1}>q_{2}\right)$. To make the benchmark case comparable to the colisting case, we let $q_{1}=1$ to model the dominant prominence of the first link on a webpage because it can capture the most user attention, just as the top organic link does in the co-listing case. Similar to the co-listing case, we denote $q_{2} \equiv 1-\psi$, where $\psi$ measures the level of information incompleteness. All other settings (i.e., firms, market preference, auction rules) follow the original model in $\S 3$.

Similarly, we start the analysis from the second stage price competition. We can formulate firms' demand functions when firm $i$ wins the first sponsored slot while the other firm $\bar{i}$ stays in the second, $\{i, \bar{i}\}=\{M, N\}$ :

$$
\begin{aligned}
& D_{i}\left(p_{i}, p_{\bar{i}}\right)=\psi A_{i}\left(p_{i}\right)+(1-\psi) S_{i}\left(p_{i}, p_{\bar{i}}\right) \\
& D_{\bar{i}}\left(p_{\bar{i}}, p_{i}\right)=(1-\psi) S_{\bar{i}}\left(p_{\bar{i}}, p_{i}\right),
\end{aligned}
$$

where $p_{i}$ and $p_{\bar{i}}$ are the firms' prices, and $A_{i}(\cdot)$ and $S_{i}(\cdot, \cdot)$ are defined by Equations (2) and (1), respectively. When firm $M$ wins the first sponsored slot and thus attracts most of the attention, as in the colisting case, the demand function is the same as before (see Equation (3)). Therefore, both firms face the same competitive situation, and the equilibrium prices and profits remain in the same format as Equation (5). The main difference, however, arises when firm $N$ wins the first sponsored slot: firm $M$ now becomes informationally disadvantaged compared to its competitor, because it no longer has the guaranteed exposure from the top organic link as in the co-listing case. In other words, the weaker firm can now win over significant informational advantage to better compete for market shares. We derive the equilibrium prices when $N$ wins the first sponsored position as follows:

$$
\left\{\begin{array}{l}
\hat{p}_{M}=\frac{2+\psi-\theta-\theta \psi}{(3+\psi)(1-\theta)} \\
\hat{p}_{N}=\frac{1+\psi+\theta-\theta \psi}{(3+\psi)(1-\theta)} .
\end{array}\right.
$$

The equilibrium profit from the second stage price competition can be derived in a similar way, as summarized by Table 3 , where $\hat{\pi}_{i}^{j}$ is firm $i^{\prime}$ s equilibrium profit in the $j$ th sponsored position in the benchmark case $(i \in\{M, N\}, j \in\{1,2\})$.

In the first stage bidding competition, both firms bid their true value: $\hat{b}_{i}=\left[\hat{\pi}_{i}^{1}-\hat{\pi}_{i}^{2}\right]^{+}(i \in\{M, N\})$, which again is the difference between the equilibrium profits when they win the first sponsored slot and when they do not. By comparing two firms' equilibrium bids $\hat{b}_{M}$ and $\hat{b}_{N}$, we can uncover the bidding outcome in the benchmark case.

Proposition 2. In the benchmark case, firm $M$ always bids higher and wins the first sponsored slot in equilibrium; that is, $\hat{b}_{M}>\hat{b}_{N}$.

Proposition 2 shows that, unlike the co-listing case in which either firm might win the sponsored bidding, the only possible bidding outcome in the benchmark case is that the firm with advantage in market preference wins the first sponsored slot. The intuition is as follows. In contrast to the co-listing case in which the differentiation in organic exposure adjusts firms' promotive and preventive incentives for sponsored bidding, in the benchmark case, winning the first sponsored slot engenders both promotive and preventive incentives equally for the two firms. The magnitudes of both incentives increase with a firm's strength in market appeal. Consequently, as long as firm $M$ has market preference advantage over $N$ (i.e., $\theta<\frac{1}{2}$ ), firm $M$ always has greater incentive to win the top sponsored slot than firm $N$.

It is also worth noting that in equilibrium, both firms adopt the same pricing strategies as in the colisting case (according to Equation (5)), and firm $M$ charges a higher price than firm $N$.

COROLlary 2. In the benchmark case, firm $N$ has a positive bidding incentive in most cases; that is, when $5 \sqrt{6} / 6-2<\theta<\frac{1}{2}, \hat{b}_{N}>0$.

Table 3 Equilibrium Profits in the Second Stage Price Competition (Benchmark Case)

\begin{tabular}{cccc}
\hline & \multicolumn{2}{c}{ When $M$ wins } & \\
\cline { 2 - 3 } & $0<\psi<\frac{1}{3}$ & $\frac{1}{3} \leq \psi<1$ & When $N$ wins \\
\hline M's profit & $\hat{\pi}_{M}^{1}=\frac{[2-\theta(1-\psi)]^{2}[(1-\theta)-(1-2 \theta) \psi]}{[3(1-\theta)(1-\psi)+4 \theta \psi]^{2}}$ & $\hat{\pi}_{M}^{1}=\left(\frac{1}{2}-\theta\right) \psi+\frac{1}{2}$ & $\hat{\pi}_{M}^{2}=\frac{(1-\psi)(2+\psi-\theta-\theta \psi)^{2}}{(3+\psi)^{2}(1-\theta)}$ \\
N's profit & $\hat{\pi}_{N}^{2}=\frac{[(1+\theta)(1-\theta)+\theta(3 \theta-1) \psi]^{2}(1-\psi)}{(1-\theta)[3(1-\theta)(1-\psi)+4 \theta \psi]^{2}}$ & $\hat{\pi}_{N}^{2}=\frac{1-\psi}{4(1-\theta)}$ & $\hat{\pi}_{N}^{1}=\frac{(1+\psi+\theta-\theta \psi)^{2}}{(3+\psi)^{2}(1-\theta)}$ \\
\hline
\end{tabular}


Compared to Corollary 1, in the benchmark case, the niche firm bids a positive amount in a larger region of parameter value. This result implies that firms' bidding incentive could be greater in the benchmark case than in the co-listing case, as we show later.

Next, we compare the equilibrium outcomes in the co-listing case with those in the benchmark case and investigate how the presence of organic listing affects social welfare, sales diversity, and search engine revenue. To focus on the systematic difference resulting from structural changes rather than the difference caused by parameter values, we let $\psi=\psi_{2}$ so that the two cases are comparable in the sense that firm $N$ receives the same level of exposure in both cases when it does not win the first sponsored slot. This parametric assumption facilitates a neat comparison and does not affect the qualitative results. We use superscript $C$ to denote the co-listing case and $B$ to denote the benchmark case.

\subsection{Effects on Social Welfare}

We consider the social welfare as the sum of total consumer surplus, both firms' profits, and the search engine's revenue. Essentially, social welfare equals the sum of the realized utility of consumers from consuming the products they have purchased. Recall that $M$ always outbids $N$ in equilibrium in the benchmark case and $M^{\prime}$ 's equilibrium price is higher than $N$ 's in both cases. We therefore can write the equilibrium social welfare as a function of information incompleteness $\psi$ in a uniform expression:

$$
\begin{aligned}
W(\theta, \psi) & \\
= & \psi\left[(1-\theta)+\theta \int_{p_{M}^{*}(\theta, \psi)}^{1} x d x\right] \\
& +(1-\psi)(1-\theta)\left(1-p_{M}^{*}(\theta, \psi)+p_{N}^{*}(\theta, \psi)\right) \\
& +(1-\psi)\left[\theta+(1-\theta) \int_{1-p_{M}^{*}(\theta, \psi)+p_{N}^{*}(\theta, \psi)}^{1} x d x\right],
\end{aligned}
$$

where $p_{M}^{*}(\theta, \psi)$ and $p_{N}^{*}(\theta, \psi)$ are defined by Equation (5). The first term represents the total realized utility of those consumers who visit firm $M^{\prime}$ 's website only and make purchases there. The second term refers to those who visit both firms' sites and buy from firm $M$, and the third term refers to those who buy from $N$ after visiting both sites. By definition, the equilibrium social welfare in the benchmark case $W^{B}=W\left(\theta, \psi_{2}\right)$. The equilibrium welfare in the co-listing case depends on the bidding outcome according to Proposition 1: $W^{C}=W\left(\theta, \psi_{2}\right)$ when $\theta<$ $\theta^{*}\left(\psi_{1}, \psi_{2}\right)$, and $W^{C}=W\left(\theta, \psi_{1}\right)$ when $\theta>\theta^{*}\left(\psi_{1}, \psi_{2}\right)$. As we can see, when $M$ wins the top sponsored slot, the welfare achieved in both cases is the same. What we are interested in is whether the presence of the organic list can increase the total social welfare when the weaker firm wins the top sponsored slot. The answer is positive.
Proposition 3. The presence of organic listing improves the social welfare in that $W^{C} \geq W^{B}$, and the strict inequality holds when $\theta>\theta^{*}\left(\psi_{1}, \psi_{2}\right)$ (defined by Equation (7)).

When $\theta>\theta^{*}\left(\psi_{1}, \psi_{2}\right), N$ wins the first sponsored slot in the co-listing case and increases its exposure level from $1-\psi_{2}$ to $1-\psi_{1}$. Proposition 3 reveals that increasing the weaker firm's exposure improves the total welfare. Note that social welfare achieves its maximum when all consumers purchase their preferred products. From Equation (10), we can identify two main sources of social efficiency loss: one is information incompleteness (characterized by the first term in Equation (10)), and the other is lack of competition (characterized by the second and third terms). When $\psi$ is high, the informational efficiency loss is high in the sense that many $N$-type consumers are not aware of product $N$ and end up buying from $M$ instead. Furthermore, a portion of $N$-type consumers who visit only M's website may leave with no purchase because of the high price charged by firm $M$. There is also competitive efficiency loss: when the degree of its informational advantage is high, firm $M$ tends to charge a high price. Consequently, a certain portion of marginal $M$-type consumers who visit both sites may purchase product $N$ rather than product $M$, which is socially inefficient. When $\psi$ is reduced, $N$ increases its exposure, and in response, $M$ charges a more competitive price in equilibrium, so both informational and competitive efficiency losses are mitigated, which results in an increase in the overall social welfare.

The comparison analysis reveals how organic listing affects social welfare: Different from the benchmark case, in the co-listing case, the significant organic exposure given to the stronger firm reduces its sponsored bidding incentive by diminishing the promotive benefit, which makes it possible for the weaker firm to win the sponsored bidding and better expose itself. In this sense, organic listing adjusts firms' relative bidding incentives by compensating the strong with extra free exposure. The outcome of such adjustment is the improvement of equilibrium information structure, which eventually increases the overall social welfare.

\subsection{Effects on Sales Diversity}

We use the Gini coefficient to measure the sales diversity. As a popular measure of inequality of income distribution, the Gini coefficient is defined as $G=1-2 \int_{0}^{1} L(x) d x$, where $L(x)$ is the Lorenz curve, which measures the lowest $100 x \%$ population's cumulative income percentage. The Gini coefficient measures the difference between the actual (income) distribution and the perfect equality/diversification case. A higher Gini coefficient indicates a greater 
degree of inequality, and a lower coefficient means greater diversification. For discrete cases, the Gini coefficient can be computed as follows:

$$
G=1-\frac{(1 / n) \sum_{i=1}^{n}\left(S_{i-1}+S_{i}\right)}{S_{n}},
$$

where $S_{i}=\sum_{j=1}^{i} y_{j}$ (and $S_{0} \equiv 0$ ), and $\left\{y_{i}\right\}_{i=1}^{n}$ is the ordered sequence of the value of interest (e.g., income) for each individual in the population such that $y_{i} \leq y_{i+1}$.

In our model, we use the Gini coefficient to measure the diversification of the realized sales across the two firms in equilibrium, and the value of interest in Equation (11) is the equilibrium sales amount. By substituting the equilibrium sales amount $D_{N}\left(p_{N}^{*}(\psi), p_{M}^{*}(\psi) ; \psi\right)$ and $D_{M}\left(p_{M}^{*}(\psi), p_{N}^{*}(\psi) ; \psi\right)$ derived from Equation (3) into Equation (11), we can calculate the Gini coefficient here as follows:

$$
\begin{aligned}
& G(\psi) \\
& \quad=\frac{1}{2}-\frac{D_{N}\left(p_{N}^{*}(\psi), p_{M}^{*}(\psi) ; \psi\right)}{D_{N}\left(p_{N}^{*}(\psi), p_{M}^{*}(\psi) ; \psi\right)+D_{M}\left(p_{M}^{*}(\psi), p_{N}^{*}(\psi) ; \psi\right)} .
\end{aligned}
$$

Similar as before, we have $G^{B}=G\left(\psi_{2}\right) ; G^{C}=G\left(\psi_{2}\right)$ when $\theta<\theta^{*}\left(\psi_{1}, \psi_{2}\right)$, and $G^{C}=G\left(\psi_{1}\right)$ when $\theta>$ $\theta^{*}\left(\psi_{1}, \psi_{2}\right)$

Proposition 4. The presence of organic listing improves the sales diversity in that $G^{C} \leq G^{B}$, and the strict inequality holds when $\theta>\theta^{*}\left(\psi_{1}, \psi_{2}\right)$ (defined by Equation (7)).

Similar to the welfare-improving effect, organic listing increases sales diversity when firm $N$ outbids firm $M$. By winning the first sponsored position under the co-listing structure, the weaker firm increases its exposure and attracts more consumers to visit its site, and among these consumers, all of the $N$-type as well as part of the $M$-type purchase from it. Therefore, the realized market share of firm $N$ is increased and the overall sales diversity is improved. In this sense, the diversity-improving effect of organic listing shares the same origin with the welfare-improving effect: compensating the strong with extra free exposure to adjust bidding incentives and to improve the equilibrium information structure.

\subsection{Effects on Search Engine Revenue}

We consider the revenue implications for the search engine from both the short-term and the longterm perspectives. We first analyze the direct effect of organic listing on search engine revenue by comparing the equilibrium revenue under both cases when holding the total consumer base fixed. More specifically, we examine the search engine's immediate revenue, $I R$, which is the revenue from sponsored bidding when the total consumer base is normalized to one as in the model setting. In secondprice auctions, the auctioneer's revenue equals the second highest bid in equilibrium. In the benchmark case, because $M$ always wins the auction, the search engine's immediate revenue $I R^{B}=\hat{b}_{N}$. In the co-listing case, $I R^{C}=b_{N}^{*}$ when $\theta<\theta^{*}\left(\psi_{1}, \psi_{2}\right)$, and $I R^{C}=b_{M}^{*}$ when $\theta>\theta^{*}\left(\psi_{1}, \psi_{2}\right)$, where $\theta^{*}\left(\psi_{1}, \psi_{2}\right)$ is defined by Equation (7).

Proposition 5. Generically, the search engine's immediate revenue is lower in the co-listing case; that is, when $5 \sqrt{6} / 6-2<\theta<\frac{1}{2}, I R^{C}<I R^{B}$ for all $0<\psi_{1}<\psi_{2}<1$.

Proposition 5 shows that compared to the case without the organic list, the presence of organic listing may reduce advertisers' bidding incentives, which leads to a lower level of immediate revenue. A brief reasoning is as follows. Because $I R^{B}=\hat{b}_{N}$ and $I R^{C}=$ $\min \left\{b_{M}^{*}, b_{N}^{*}\right\}$, to conclude that $I R^{B}>I R^{C}$, it is sufficient to show that $\hat{b}_{N}>b_{N}^{*}$. Recall that a firm's equilibrium bid equals the difference between its equilibrium profits when winning the top sponsored slot and when not winning it (i.e., according to Tables 2 and $3, b_{N}^{*}=$ $\pi_{N}^{*}\left(\psi_{1}, \theta\right)-\pi_{N}^{*}\left(\psi_{2}, \theta\right)$ and $\left.\hat{b}_{N}=\hat{\pi}_{N}^{1}-\hat{\pi}_{N}^{2}\right)$. On the one hand, as discussed earlier, when $N$ does not win the top sponsored slot, the equilibrium profit achieved in both cases is the same (i.e., $\left.\pi_{N}^{*}\left(\psi_{2}, \theta\right)=\hat{\pi}_{N}^{2}\right)$. On the other hand, when $N$ wins the top sponsored slot, in the co-listing case, $M$ still possesses significant exposure from the organic list, which limits $N^{\prime}$ s profit because of the consequent intense price competition. In the benchmark case, however, $N$ could overturn the informational dominance structure thoroughly and greatly improve the profitability of winning the top sponsored slot. As a result, $N^{\prime}$ s winning profit in the benchmark case is higher in general (i.e., $\hat{\pi}_{N}^{1}>$ $\pi_{N}^{*}\left(\psi_{1}, \theta\right)$ ). In consequence, as long as $\hat{b}_{N}>0$ (i.e., under a certain boundary condition on $\theta$ according to Corollary 2), we can conclude that $\hat{b}_{N}>b_{N}^{*}$ and hence $I R^{B}>I R^{C}$. It is also worth noting that in addition to $N^{\prime}$ s decreased bidding incentive, firm $M^{\prime}$ 's incentive to bid for a sponsored link is also less under the co-listing case than under the benchmark case, sometimes even to a greater degree such that firm $M$ might lose the bidding competition to firm $N$ in the co-listing case.

The driving force of this result is the extra free exposure from the organic list. Because of the significant organic exposure the stronger firm always possesses, winning the top sponsored position is not so profitable for the weaker firm, and losing the bidding competition is not too bad for the stronger firm, which leads to lower bidding incentives for both firms in presence of organic listing. In this sense, although the differentiated organic exposure adjusts firms' relative 
bidding incentive in a way favoring the social welfare and the sales diversity, the extra organic exposure given to the stronger firm lowers both firms' bidding incentives in absolute terms and might hurt the search engine's immediate revenue.

Note that Proposition 5 analyzes the search engine revenue under the monopolistic search engine setting such that the consumer base is assumed to be fixed. In general, when outside options are available (e.g., competing search engines exist), a search engine's consumer base may change as it adopts different designs, which might affect its revenue in the long run. Therefore, we are also interested in how the presence of organic listing may affect the search engine's consumer base. In particular, we are interested in how the equilibrium consumer surplus differ under the benchmark case and the co-listing case. The equilibrium consumer surplus, $C S$, is defined as follows:

$$
\begin{aligned}
\operatorname{CS}(\theta, \psi) \\
=\psi\left[(1-\theta)\left(1-p_{M}^{*}(\theta, \psi)\right)+\theta \int_{p_{M}^{*}(\theta, \psi)}^{1}\left(x-p_{M}^{*}(\theta, \psi)\right) d x\right] \\
\quad+(1-\psi)(1-\theta)\left(1-p_{M}^{*}(\theta, \psi)+p_{N}^{*}(\theta, \psi)\right) \\
\quad \cdot\left(1-p_{M}^{*}(\theta, \psi)\right)+(1-\psi)\left[\theta\left(1-p_{N}^{*}(\theta, \psi)\right)\right. \\
\left.\quad+(1-\theta) \int_{1-p_{M}^{*}(\theta, \psi)+p_{N}^{*}(\theta, \psi)}^{1}\left(x-p_{N}^{*}(\theta, \psi)\right) d x\right],
\end{aligned}
$$

where $p_{M}^{*}(\theta, \psi)$ and $p_{N}^{*}(\theta, \psi)$ are equilibrium prices as before. Equation (13) can be understood in a similar fashion as the definition of social welfare in Equation (10). By definition, $\operatorname{CS}^{B}=C S\left(\theta, \psi_{2}\right)$, and $C S^{C}=$ $\operatorname{CS}\left(\theta, \psi_{1}\right)$ when $\theta>\theta^{*}\left(\psi_{1}, \psi_{2}\right)$.

Note that Equation (13) equals the expected net utility of any individual customer before entering the search market (without knowing her actual preference before the search). In this sense, if we consider that consumers have different reserve utilities ${ }^{3}$ and that they use this particular search engine if the expected utility exceeds their reserve values and leave for outside options otherwise, then the total consumer volume attracted to the search engine can be viewed as an increasing function of CS. As we are interested in the general trends rather than in the detailed dynamics, comparing the equilibrium consumer surplus under the two cases can deliver essential implications for the effect of organic listing on the growth of the search engine's consumer base in the long run.

Proposition 6. The equilibrium consumer surplus in the co-listing case is higher in that $C S^{C} \geq C S^{B}$, and the strict inequality holds when $\theta>\theta^{*}\left(\psi_{1}, \psi_{2}\right)$ (defined by Equation (7)).

\footnotetext{
${ }^{3}$ Such different reserve utilities can result from a combination of search costs, switching costs, preference over different options, and expected net utilities that other options offer.
}

When the organic list is present, similar to the effects on social welfare, the differentiated organic exposure dilutes firm $M^{\prime}$ 's bidding incentive and gives firm $N$ more chances to win a better sponsored position for a better exposure. As a result, consumers are more likely to find the product they prefer-at lower prices because of the intensified price competition. Therefore, by inducing a lower level of information incompleteness $\psi$ in equilibrium, the co-listing case mitigates both the informational and competitive loss of consumer surplus, which improves the overall consumer surplus.

Combining the results from Propositions 5 and 6, we can conclude the revenue implication for search engine: organic listing trades off immediate revenue for higher consumer surplus, which could lead to a growing consumer base. In this sense, organic listing serves as a balance between short-term profitability and long-term growth. If the search engine values the long-term benefit enough, then organic listing could actually be beneficial for revenue growth. In this sense, the co-listing structure reflects certain far-sighted wisdom.

To summarize, in this section, we compare the equilibrium outcomes in the co-listing case with those in a benchmark case. We show that although the presence of organic listing may reduce advertisers' bidding incentives, it adjusts the bidding outcome so that the equilibrium social welfare, sales diversity, and consumer surplus can all be improved. Meanwhile, it is worth pointing out that these beneficial effects of organic listing function well only under moderate levels of market asymmetry (i.e., firms are comparable to each other in market appeal, or $\theta$ is not too small). Nevertheless, these effects malfunction when the competence difference among advertisers is too large to adjust. For firms at the very tail end of the distribution (i.e., for weak firms with very small $\theta$ ), even under the co-listing structure, their bidding incentive is still lower than that of the strong competitors. As a result, the strong firms occupy the top positions in both lists, and no fundamental change occurs in information structure after the sponsored bidding competition. Consequently, while bearing potential loss in revenue, the search engine cannot engender structural improvement in social welfare, sales diversity, and consumer surplus. In this sense, the typical organic listing design serves the middles well, rather than the tails.

\section{Extension and Discussion}

\subsection{Improving Organic Ranking}

The previous analysis pinpoints the malfunction of organic listing's beneficial effects for highly asymmetric markets (i.e., when $\theta$ is very small). In this section, 
we explore the possibility to mitigate such drawbacks so that organic listing can serve the tails better.

We have shown that organic listing improves social welfare and consumer surplus by giving the stronger firm more free exposure to adjust firms' relative bidding incentive and to help the weaker firm win a better sponsored position. Nevertheless, the extra organic exposure given to the stronger firm also adversely hurts the weaker firm's bidding incentive, especially when it is highly disadvantaged in market appeal. Given such a trade-off, slightly reducing the organic exposure difference among firms might lead to favorable outcomes for highly asymmetric markets. Along this line, we may consider promoting the weak in the organic list by introducing perturbation or uncertainty in firms' organic ranks. Instead of ranking firms in an order based strictly on their strength or popularity, which we refer to as pure organic ranking, we may consider a mixed organic ranking mechanism in which the organic list ranks firms by their popularity with certain probability and in an inverse order otherwise. Mixed ranking can be implemented by simply randomizing the ordering of targeted advertisers with certain probability, or by including additional factors other than the popularity measure into the organic ranking rule.

We illustrate the idea of mixed organic ranking by slightly modifying the model. Following the model setup in $\S 3$, we consider a mixed organic ranking in which with probability $1-\lambda(0 \leq \lambda \leq 1)$, firm $M$ is listed in a top organic slot on the SERP, and firm $N$ is listed in a less-prominent organic slot. Meanwhile, with probability $\lambda$, firm $N$ is given a top organic position, and $M$ stays in a less-prominent organic position. As a result, when $M$ wins the first sponsored slot, the probability of $M$ being visited by a consumer is $(1-\lambda)+\lambda\left(1-\psi_{1}\right)=1-\lambda \psi_{1}$, and the probability of $N$ being visited is $\lambda+(1-\lambda)\left(1-\psi_{2}\right)=$ $1-(1-\lambda) \psi_{2}$. Similarly, when $N$ wins the first sponsored slot, the probability of $N$ being visited and that of $M$ being visited are $1-(1-\lambda) \psi_{1}$ and $1-\lambda \psi_{2}$, respectively. The pure organic ranking is thus a special case of mixed ranking with $\lambda=0$. To simplify the discussion, we let $\psi_{1}=0$ because the first sponsored link often attracts considerable attention such that $\psi_{1}$ is small in general. Results remain qualitatively the same for positive $\psi_{1}$.

We can formulate firms' demand functions similarly as before. When firm $M$ wins the first sponsored slot, $M$ possesses informational monopoly power only if $N$ is placed in the less prominent organic slot (with probability $1-\lambda$ ) and is not noticed by a consumer (with probability $\psi_{2}$ ). Therefore, the demand functions facing both firms are as follows:

$$
\begin{aligned}
D_{M}^{1}\left(p_{M}, p_{N}\right)= & (1-\lambda) \psi_{2} A_{M}\left(p_{M}\right) \\
& +\left[1-(1-\lambda) \psi_{2}\right] S_{M}\left(p_{M}, p_{N}\right) \\
D_{N}^{2}\left(p_{N}, p_{M}\right)= & {\left[1-(1-\lambda) \psi_{2}\right] S_{N}\left(p_{N}, p_{M}\right), }
\end{aligned}
$$

where $A_{i}(\cdot)$ and $S_{i}(\cdot)$ are defined as before by Equations (1) and (2). When firm $N$ wins the sponsored bidding, similarly, the demand functions become

$$
\begin{aligned}
& D_{M}^{2}\left(p_{M}, p_{N}\right)=\left(1-\lambda \psi_{2}\right) S_{M}\left(p_{M}, p_{N}\right) \\
& D_{N}^{1}\left(p_{N}, p_{M}\right)=\lambda \psi_{2} A_{N}\left(p_{N}\right)+\left(1-\lambda \psi_{2}\right) S_{N}\left(p_{N}, p_{M}\right) .
\end{aligned}
$$

Note that by introducing probabilistic alteration in organic ranking, the strong firm no longer possesses guaranteed informational advantage, and in some instances, the weak firm is able to gain informational advantage once it wins the top sponsored position.

Along a similar approach as before, we can derive the equilibrium prices $\tilde{p}_{i}^{j}$ and equilibrium profits $\tilde{\pi}_{i}^{j}$ for firm $i$ in the $j$ th sponsored position, where $i \in$ $\{M, N\}$ and $j \in\{1,2\}$. The equilibrium bidding, therefore, is $\tilde{b}_{i}=\left[\Delta \tilde{\pi}_{i}\right]^{+}$, where $\Delta \tilde{\pi}_{i}=\tilde{\pi}_{i}^{1}-\tilde{\pi}_{i}^{2}$.

We are interested to see whether introducing mixed organic ranking can improve the equilibrium outcomes. Therefore, we focus on the effect of a slight perturbation of the pure organic ranking by examining the marginal effect of increasing $\lambda$ evaluated at $\lambda=0$. Note that when $\lambda=0$, the equilibrium outcomes are the same as in the original model with the pure organic ranking. We can derive the equilibrium social welfare $\tilde{W}$, Gini coefficient $\tilde{G}$, and consumer surplus $\widetilde{C S}$ in a similar fashion. For example, the equilibrium social welfare can be written as

$$
\begin{aligned}
\tilde{W}\left(\theta, \psi_{2}, \lambda\right) \\
=(1-\lambda) \psi_{2}\left[(1-\theta)+\theta \int_{\tilde{p}_{M}^{1}}^{1} x d x\right] \\
\quad+\left[1-(1-\lambda) \psi_{2}\right](1-\theta)\left(1-\tilde{p}_{M}^{1}+\tilde{p}_{N}^{2}\right) \\
\quad+\left[1-(1-\lambda) \psi_{2}\right]\left[\theta+(1-\theta) \int_{1-\tilde{p}_{M}^{1}+\tilde{p}_{N}^{2}}^{1} x d x\right] .
\end{aligned}
$$

Recall that in the case when $\theta$ is small and $\psi_{2}$ is not too large, pure organic ranking engenders low revenue from sponsored bidding with little improvement in consumer surplus, social welfare, or sales diversity. The next result shows that, under this particular case, introducing mixed organic ranking could be beneficial in these aspects.

Proposition 7. For highly asymmetric markets, introducing mixed organic ranking can improve social welfare, sales diversity, consumer surplus, and search engine's immediate revenue concurrently in that when $\theta<\theta_{0}$ and $\psi_{2}<1 /(3(1-\lambda))$, (i) $\partial \tilde{W}\left(\theta, \psi_{2}, \lambda\right) /\left.\partial \lambda\right|_{\lambda=0}>0$; 
(ii) $\partial \tilde{G}\left(\theta, \psi_{2}, \lambda\right) /\left.\partial \lambda\right|_{\lambda=0}<0$; (iii) $\partial \widetilde{C S}\left(\theta, \psi_{2}, \lambda\right) /\left.\partial \lambda\right|_{\lambda=0}$ $>0$; and (iv) $\partial \Delta \tilde{\pi}_{N}\left(\theta, \psi_{2}, \lambda\right) /\left.\partial \lambda\right|_{\lambda=0}>0$. Here, $\theta_{0} \in\left(0, \frac{1}{2}\right)$ solves the equation $\partial \Delta \tilde{\pi}_{N}\left(\theta_{0}, \psi_{2}, \lambda\right) /\left.\partial \lambda\right|_{\psi_{2}=1 /(3(1-\lambda)), \lambda=0}=0$.

Under pure organic ranking, as is implied by Corollary 1 , when $\theta$ is small and $\psi_{2}$ is not too large, firm $N$ 's promotive incentive for winning the top sponsored position completely vanishes because of its huge disadvantage in market preference and $M^{\prime}$ 's unshakable informational advantage in the organic list, which hurts the revenue contributed to the search engine. In contrast, mixed organic ranking probabilistically reduces $M^{\prime}$ s informational advantage in the organic list, which adds preventive incentive to $N$ 's sponsored bidding motivation. As the weak firm becomes more comparable in terms of exposure, its bidding incentive increases.

On the other hand, the equilibrium consumer surplus and social welfare are improved as well, because mixed ranking reduces the aforementioned two sources of efficiency loss. The perturbation in organic rank directly improves firm $N$ 's exposure and thus effectively reduces the informational loss of social welfare and consumer surplus. Meanwhile, a smaller difference in exposure level induces more intense price competition so that the competitive efficiency loss is also mitigated.

An interesting aspect of Proposition 7 is that welfare and revenue can be improved simultaneously, unlike in most existing studies in which increasing revenue is often at the cost of welfare. The key driver to this result is the self-balance between organic ranking and sponsored bidding. Although mixed ranking reduces firm $M^{\prime}$ 's organic exposure, it does not significantly decrease $M^{\prime}$ 's overall exposure because $M$ regains substantial exposure by winning the top sponsored position. Therefore, mixed ranking promotes the weak firm's exposure at little cost to the strong's, which leads to less informational efficiency loss in total and thus higher consumer surplus and social welfare. Therefore, mixed organic ranking allocates the total resource of consumer attention in a more effective way.

It is worth noting that our focus is on how information structure affects firms' strategies and the equilibrium outcomes, and hence we discuss the benefits of introducing mixed organic ranking from this particular perspective. Nevertheless, when implementing in practice, we also need to consider other factors that are outside the scope of the model. For example, while we show that mixed organic ranking can help some consumers find the product they prefer and increase the total consumer surplus, the uncertainty in ordering the organic results may risk the search engine's objectivity and reliability perceived by the general users who search only for noncommercial information from the organic results. In addition, although mixed organic ranking is designed to be an impartial modification of the ranking mechanism to improve the overall performance rather than a manipulation against any side, major advertisers might feel that they are unfairly treated and that their interests are hurt. Therefore, the scale and scope of mixing need to be chosen with special caution so that the alteration in organic ranking is limited to certain commercial websites in certain markets and it does not impair the utility of general search engine users. Meanwhile, additional incentives could be provided to strong advertisers in the sponsored auction rules. The discussion here serves as the first step in exploring the optimal design of organic ranking mechanism.

\subsection{General Information Structure}

In the previous analysis, we keep the model simple to derive clear implications while capturing the essence of interest. In this section, we extend the model to a more general framework that allows more flexible parameter values for different information structures. As we show, the main results continue to hold in various scenarios under this framework.

So far we have assumed that firm M's website is visited by consumers with probability 1 regardless of its sponsored position because of the dominant advantage of its top organic position (i.e., $\alpha_{i_{M}}=1$ ). Now we relax this assumption by considering that firm $M^{\prime}$ 's website is visited with probability $1-\psi_{1}^{\prime}$ when $M$ wins the first sponsored position, and with probability $1-\psi_{2}^{\prime}$ when it stays in the second $\left(0<\psi_{1}^{\prime}<\right.$ $\left.\psi_{2}^{\prime}<1\right)$. We continue to assume that firm $N^{\prime}$ 's website is visited with probability $1-\psi_{1}$ when $N$ wins the first sponsored position, and with probability $1-\psi_{2}$ when $N$ stays in the second sponsored position $\left(0<\psi_{1}<\right.$ $\left.\psi_{2}<1\right)$. Because the organic list gives the stronger firm more exposure, firm $M$ would attain higher attention level than firm $N$ should they stay in the same sponsored position, and therefore, $\psi_{1}^{\prime}<\psi_{1}$ and $\psi_{2}^{\prime}<\psi_{2}$. In the benchmark case in which there is only a sponsored list, firm's sponsored position determines their overall exposure, and therefore, $\psi_{1}=\psi_{1}^{\prime}$ and $\psi_{2}=\psi_{2}^{\prime}$.

Given $M^{\prime}$ 's and $N^{\prime}$ s exposure levels, which are characterized by $\psi^{\prime}$ and $\psi$, respectively, their demand functions can be formulated as follows:

$$
\begin{aligned}
& \quad D_{M}\left(p_{M}, p_{N}\right) \\
& \quad=\left(1-\psi^{\prime}\right)\left[\psi A_{M}\left(p_{M}\right)+(1-\psi) S_{M}\left(p_{M}, p_{N}\right)\right] \\
& D_{N}\left(p_{N}, p_{M}\right) \\
& \quad=(1-\psi)\left[\psi^{\prime} A_{N}\left(p_{N}\right)+\left(1-\psi^{\prime}\right) S_{N}\left(p_{N}, p_{M}\right)\right],
\end{aligned}
$$


where the $S_{i}(\cdot, \cdot)$ and $A_{i}(\cdot)$ functions follow the same definition as in Equations (1) and (2). Following a similar approach as before, we can solve firms' equilibrium prices as follows:

$$
\left\{\begin{array}{l}
p_{M}^{*}=\min \left\{\frac{2-(1-\psi) \theta+\psi^{\prime}(1-\psi)(1-\theta)}{3(1-\psi)(1-\theta)+4 \psi \theta+\psi^{\prime}(1-\psi)(1-\theta)}, 1\right\} \\
p_{N}^{*}=\frac{\theta+(1-\theta) \psi^{\prime}+(1-\theta)\left(1-\psi^{\prime}\right) p_{M}^{*}}{2(1-\theta)} .
\end{array}\right.
$$

Note that when $\psi^{\prime}=0$, Equations (17) and (18) are reduced to the original results in Equations (3) and (5). Substituting the equilibrium prices back into firms' respective profit functions, we can derive the equilibrium profit functions $\pi_{i}^{*}\left(\theta, \psi, \psi^{\prime}\right), i \in\{M, N\}$.

Firms' bidding incentives are determined by the profit difference between winning the first sponsored position and not; that is, $b_{i}=\left[\Delta \pi_{i}\right]^{+}$, where $\Delta \pi_{M}=\pi_{M}^{*}\left(\theta, \psi_{2}, \psi_{1}^{\prime}\right)-\pi_{M}^{*}\left(\theta, \psi_{1}, \psi_{2}^{\prime}\right)$ and $\Delta \pi_{N}=$ $\pi_{N}^{*}\left(\theta, \psi_{1}, \psi_{2}^{\prime}\right)-\pi_{N}^{*}\left(\theta, \psi_{2}, \psi_{1}^{\prime}\right)$. For a better understanding of how the organic listing impacts the sponsored bidding, we can rewrite $\Delta \pi_{M}$ and $\Delta \pi_{N}$ as follows:

$$
\begin{aligned}
\Delta \pi_{M}= & {\left[\pi_{M}^{*}\left(\theta, \psi_{2}, \psi_{1}^{\prime}\right)-\pi_{M}^{*}\left(\theta, \psi_{1}, \psi_{1}^{\prime}\right)\right] } \\
& +\left[\pi_{M}^{*}\left(\theta, \psi_{1}, \psi_{1}^{\prime}\right)-\pi_{M}^{*}\left(\theta, \psi_{1}, \psi_{2}^{\prime}\right)\right] \\
\Delta \pi_{N}= & {\left[\pi_{N}^{*}\left(\theta, \psi_{1}, \psi_{2}^{\prime}\right)-\pi_{N}^{*}\left(\theta, \psi_{1}, \psi_{1}^{\prime}\right)\right] } \\
& +\left[\pi_{N}^{*}\left(\theta, \psi_{1}, \psi_{1}^{\prime}\right)-\pi_{N}^{*}\left(\theta, \psi_{2}, \psi_{1}^{\prime}\right)\right] .
\end{aligned}
$$

As we can see, the terms in the first bracket of Equations (19) and (20) account for the preventive incentive for firm $M$ and firm $N$, respectively, and the terms in the second bracket account for their promotive incentive, because, for example, $\pi_{M}^{*}\left(\theta, \psi_{2}, \psi_{1}^{\prime}\right)-$ $\pi_{M}^{*}\left(\theta, \psi_{1}, \psi_{1}^{\prime}\right)$ measures the difference in $M^{\prime}$ 's equilibrium profits between letting $N$ have a lower exposure level (i.e., $1-\psi_{2}$ ) versus a higher exposure level (i.e., $1-\psi_{1}$ ) while holding $M^{\prime}$ 's own exposure level unchanged (i.e., $1-\psi_{1}^{\prime}$ ). Recall that in the original model, $\psi_{1}^{\prime}=\psi_{2}^{\prime}=0$, and thus the terms in the second bracket of Equation (19) and in the first bracket of Equation (20) vanish, which explains why firm $M$ has preventive incentive only, and $N$ has promotive incentive only. In contrast, in this general framework, either firm has both promotive and preventive incentives. Nevertheless, the relative strengths of the two incentives are different for the two firms because of the presence of the organic list. As the organic list differentiates firms' organic exposure according to their market strength, the higher level of organic exposure given to the stronger firm reduces the marginal benefit of winning a better sponsored position in increasing the overall exposure level. Reflected in the parameter values, the difference between $\psi_{2}^{\prime}$ and $\psi_{1}^{\prime}$ should be smaller than that between $\psi_{2}$ and $\psi_{1}$. As a result, M's preventive incentive $\left(\pi_{M}^{*}\left(\theta, \psi_{2}, \psi_{1}^{\prime}\right)-\pi_{M}^{*}\left(\theta, \psi_{1}, \psi_{1}^{\prime}\right)\right)$ outweighs its promotive incentive $\left(\pi_{M}^{*}\left(\theta, \psi_{1}, \psi_{1}^{\prime}\right)-\pi_{M}^{*}\left(\theta, \psi_{1}, \psi_{2}^{\prime}\right)\right)$, whereas the promotive aspect dominates $N^{\prime}$ s bidding incentive. The dominating parts play a major role in comparing firm's bidding incentives, so the bidding outcome and the equilibrium results follow patterns similar to those in the original model.

Given the equilibrium pricing and bidding, the social welfare, the consumer surplus, and the Gini coefficient for sales diversity can be derived in a similar fashion. For example, the equilibrium social welfare under the general framework equals

$$
\begin{aligned}
W\left(\theta, \psi, \psi^{\prime}\right)= & \psi\left(1-\psi^{\prime}\right)\left[1-\theta+\theta \int_{p_{M}^{*}}^{1} x d x\right] \\
& +(1-\psi) \psi^{\prime}\left[\theta+(1-\theta) \int_{p_{N}^{*}}^{1} x d x\right] \\
& +(1-\psi)\left(1-\psi^{\prime}\right)\left[(1-\theta)\left(1-p_{M}^{*}+p_{N}^{*}\right)\right. \\
& \left.+\theta+(1-\theta) \int_{1-p_{M}^{*}+p_{N}^{*}}^{1} x d x\right] .
\end{aligned}
$$

The following examples illustrate the equilibrium outcomes under the general framework.

EXAMPLE 1. Let $\psi_{1}=0.10, \psi_{2}=0.30, \psi_{1}^{\prime}=0.05$, and $\psi_{2}^{\prime}=0.08$. In equilibrium, there exists a cutoff $\theta^{*}=$ 0.386 such that when $\theta<\theta^{*}, M$ bids higher than $N$; when $\theta>\theta^{*}, N$ outbids $M$. Compared to the benchmark case without the organic list, the co-listing case results in higher social welfare, sales diversity, and consumer surplus when $\theta>\theta^{*}$, but a lower immediate revenue for the search engine in general (i.e., when $\theta>0.034)$. Figure 1 illustrates firms' bidding incentives under both the co-listing and the benchmark cases.

In both Figures 1(a) and 1(b), the dashed lines represent firms' preventive incentives, the dash-dotted lines represent firms' promotive incentives, and the solid lines represent firms' overall bidding incentives. As we can see, in the co-listing case, $M^{\prime}$ 's preventive incentive accounts for the majority of its total bidding incentive, while N's promotive incentive closely approximates its overall bidding incentive, and the two firms' bidding incentive curves intersect at the cutoff $\theta^{*}$. In contrast, in the benchmark case, firm $M^{\prime}$ 's preventive and promotive incentives are both greater than $N^{\prime}$ s, resulting in $M^{\prime}$ 's bidding incentive being always higher than $N$ 's.

Similar to the original model, Example 1 represents a typical case in which the top organic position attracts the most attention: firm $M$ possesses predominant advantage in total attention even if it does not win the first sponsored position; that is, 
Figure 1

Illustration of Bidding Incentives in Example 1

(a) The co-listing case

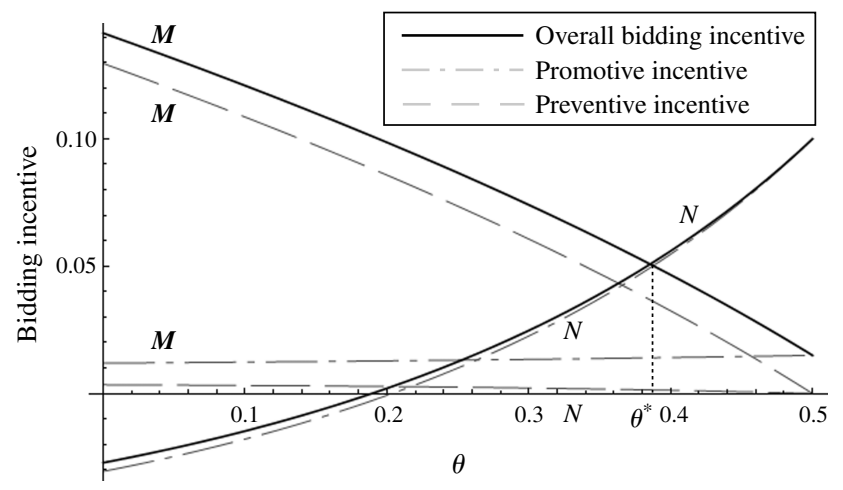

(b) The benchmark case

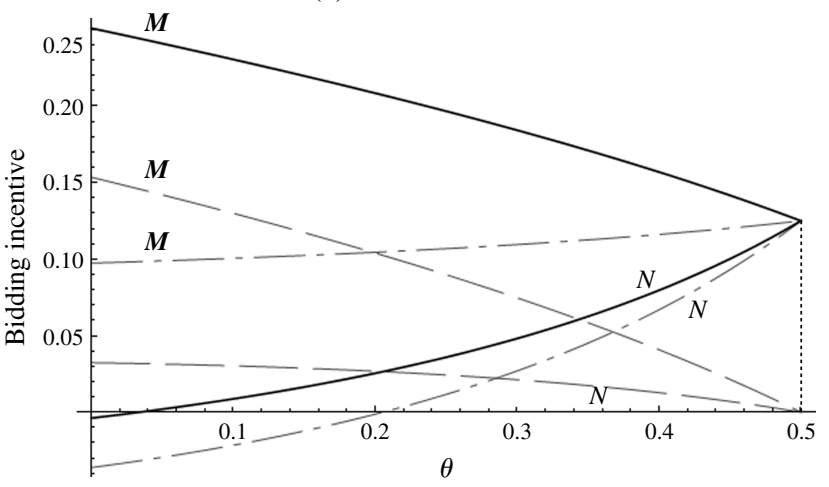

$1-\psi_{2}^{\prime}>1-\psi_{1}$, or equivalently, $\psi_{2}^{\prime}<\psi_{1}$. It is worth noting that in the practice, search engines often display premium sponsored links in a highlighted area in the top central part of the SERP. Although consumers still tend to click the top organic links more than these premium sponsored links because of the aversion to advertisement in general (Lutz 1985), we are also interested to see how the results can extend to the case in which sponsored positions have higher probability of being clicked than organic positions.

EXAMPLE 2. Let $\psi_{1}=0.05, \psi_{2}=0.30, \psi_{1}^{\prime}=0.03$, and $\psi_{2}^{\prime}=0.20$. In equilibrium, there exists a cutoff $\theta^{*}=$ 0.457 such that when $\theta<\theta^{*}, M$ bids higher than $N$; when $\theta>\theta^{*}, N$ outbids $M$. Compared to the benchmark case without the organic list, the co-listing case achieves higher social welfare, sales diversity, and consumer surplus when $\theta>\theta^{*}$, but lower immediate revenue for the search engine in general (i.e., when $\theta>0.031)$.

Example 2 represents the case in which a firm's overall exposure level is determined primarily by its sponsored position rather than its organic position. In this case, $N$ can achieve significant exposure level if it wins the first sponsored position; that is, $1-\psi_{1}$ can be very close to $1-\psi_{1}^{\prime}$ and considerably higher than
$1-\psi_{2}^{\prime}\left(M^{\prime}\right.$ s exposure level when it stays at the second sponsored position). As Example 2 illustrates, the equilibrium outcomes remain qualitatively the same as before. Compared to Example 1, the cutoff point $\theta^{*}$ in Example 2 shifts toward $\frac{1}{2}$, suggesting that the weak firm is less likely to win the sponsored bidding as the effect of organic listing decreases.

\subsection{Multiple Competing Firms}

Throughout the paper, we base our analysis on a duopoly setting given the essential features of the focal problem, such as the multidimensional asymmetry among competing firms and the endogenous valuation of the auction objects. In this section, we briefly discuss possible extensions to a general case involving multiple competing firms. In a general oligopoly setting, firms with different market appeal and different exposure solve their respective optimization problems in pricing, resulting in different valuations of sponsored positions across firms, which further differ over each possible allocation of sponsored positions and thus complicate the bidding competition. For this reason, equilibrium analysis under general oligopolistic competition is analytically intractable. We next consider a particular type of oligopolistic competition to illustrate how the analysis and results can be extended beyond the duopoly case. Because of the page limit, we present the details in the online appendix (available at http://dx.doi.org/ 10.1287 /isre.1120.0425).

In brief, we consider a case in which one strong firm, whose product is preferred by the mainstream market, competes against multiple (symmetric) weak firms, whose products are designed for particular niche markets that are mutually exclusive to each other. Because of the differentiated exposure in the organic list aligned with firms' market appeal, the strong firm possesses a predominant advantage in exposure regardless of its sponsored position, whereas weak firms increase their exposure by improving their sponsored rank. Such a structure allows us to derive the equilibrium pricing explicitly. In determining the bidding equilibrium, we consider the particular type of locally envy-free equilibrium studied by Edelman et al. (2007), in which each firm bids an amount equal to its own payment plus its own profit difference between staying in the current sponsored position and moving one position up. We show that when the market preference difference between the strong firm and the weak firms is not too large, all weak firms bidding higher than the strong firm is an equilibrium, which resembles the bidding outcomes in the duopoly case. The driving force is the similar interplay between promotive and preventive bidding incentives. With the presence of organic listing, firms' incentives for acquiring a better sponsored 
position are altered. Moving up its sponsored rank directly benefits a weak firm by increasing its exposure, whereas a better sponsored position benefits the strong firm only in that it blocks one of the competitors to a greater degree. The former promotive incentive grows to exceed the latter preventive incentive, as the weak firms' market appeal strengthens. As a result, the profit gain from moving up one position in the sponsored list for the weak firms exceeds that for the strong firm, and therefore, all weak firms bid higher than the strong firm in equilibrium.

\section{Conclusion}

In studying the role of organic listing in search advertising, we focus on the effects of organic listing as a competing information source on advertisers' sponsored bidding incentives and the equilibrium outcomes. This paper thus provides implications for both search engine designers and marketing managers.

First, we provide economic justification for the common practice of the co-listing structure in the search industry. As we demonstrate, the organic list not only serves as an objective list providing reliable information to general information seekers, but also positively affects the sponsored bidding to improve the overall information structure that the search engine provides. On the surface, organic listing gives the advertiser stronger in market appeal more free exposure. In effect, such differentiated allocation of free exposure adjusts advertisers' relative bidding incentives to give the weaker advertiser more chances to win a better sponsored position, which facilitates consumers to locate the products they prefer and therefore increases the consumer surplus, social welfare, and sales diversity. Although the presence of the free exposure from the organic list may reduce advertisers' sponsored bidding incentive per se, the overall effect benefits the search engine's growth in the long run.

Meanwhile, we also explore a possible direction to improve the organic ranking mechanism for certain scenarios. As we pinpoint, the beneficial effects of organic listing function well when advertisers are relatively comparable in market appeal. For highly asymmetric markets in which huge competence difference exists among advertisers, introducing slight perturbation in organic ranking could increase the weaker advertiser's sponsored bidding incentive as well as social welfare and consumer surplus. Possible implementations include randomizing the organic ranks of targeted advertisers with certain probability, or incorporating additional factors with uncertainty (e.g., third-party reviews) into the organic ranking score. On the other hand, caution needs to be taken in determining the scale and scope of such perturbation to avoid possible misperception of general users and advertisers. The optimal design of organic ranking mechanism thus calls for further study.

In addition to the implications for search engine designers, our study also provides rationale for marketing managers in dealing with online search advertising. Advertisers whose websites have already been placed in top organic positions may wonder whether it is still worth investing in sponsored bidding. We suggest that sponsored bidding is rewarding when advertisers have a salient advantage in market preference, and dominating exposure in both lists maximizes their profitability. When market preference is relatively diversified, advertisers with unsatisfactory organic rank should bid aggressively to win the top sponsored slots because the marginal benefit of increasing sponsored exposure is fairly high in this case. In contrast, for very niche firms or individual sellers without sufficient market appeal, although it may seem tempting to promote themselves via this new advertising channel, excessive spending in sponsored bidding may have a low return on investment, because even a very prominent sponsored position with a high click-through rate may bring high advertising bills but not commensurable profits.

This paper has several limitations, which trigger directions for future research. First, we do not model the competition among search engines, and our analysis is limited to a monopolistic search engine. Results on effects of altering organic listing could be sensitive to competition among search engines. Although we discuss the consumer surplus in the paper, which, to a certain extent, captures consumers' reactions when an outside option is available, explicitly modeling search engine competition would more directly reflect the strategic interactions among search engines. Further along the line of search engine competition, there arise other interesting directions for future research. For example, we may consider different revenue model choices in addition to advertising when search engines compete against each other, which could link the study on search advertising to another stream of research on the business model choice of competing firms (e.g., Casadesus-Masanell and Zhu 2010, 2012). Second, our analysis is primarily based on duopoly advertisers. Although we extend the results to multiple competing firms under certain cases, the general oligopolistic analysis is intractable under our framework. Because the competition among multiple advertisers can provide a better approximation of the reality and richer implications regarding the competition outcomes, how to study the asymmetric competition in which multiple advertisers bid for multiple advertising positions with the information structure aligned with the market structure is thus a challenging yet intriguing question for future research. 


\section{Electronic Companion}

An electronic companion to this paper is available as part of the online version at http://dx.doi.org/10.1287/ isre.1120.0425.

\section{Acknowledgments}

The authors thank the senior editor, the associate editor, and two anonymous reviewers for their very helpful comments, which greatly improved this paper. The authors also thank Thomas A. Weber for his generous input. The authors thank the seminar participants at The University of Texas at Austin, University of Arizona, Georgia Institute of Technology, University of Florida, The Hong Kong University of Science and Technology, University of Hong Kong, the Fourteenth Conference on Information Systems and Technology (2009), INFORMS Annual Meeting (2010), and The Workshop on Information Systems Economics (2010) for their helpful feedback. A previous version of this article was circulated under the title "Too Organic for Organic Listing? Interplay between Organic and Sponsored Listing in Search Advertising."

\section{Appendix. Proofs}

Because of the page limit, we provide the outline of all proofs only. Detailed proofs are available upon request.

Proof of Lemma 1. According to Table 2, (i) If $\frac{1}{3} \leq$ $\psi<1$, then $\partial^{2} \pi_{M}^{*}(\theta, \psi) / \partial \theta \partial \psi=-1<0$ and $\partial^{2} \pi_{N}^{*}(\theta, \psi) / \partial \theta \partial \psi$ $=-1 /\left(4(1-\theta)^{2}\right)<0$.

(ii) If $0<\psi<\frac{1}{3}$, then $\partial^{2} \pi_{M}^{*}(\theta, \psi) / \partial \theta \partial \psi=f(\psi, \theta) /(3-3 \theta-$ $3 \psi+7 \theta \psi)^{4}$, where $f(\psi, \theta)$ is a polynomial function of $\psi$ and $\theta$ with degree of $8\left(\psi^{4} \theta^{4}\right)$. We can show that $f(\psi, \theta)$ is convex in $\psi$ for $0<\psi<\frac{1}{3}$ and $0<\theta<\frac{1}{2}$. We can verify that $f(0, \theta)<0$ and $f\left(\frac{1}{3}, \theta\right)<0$ for $\forall \theta \in\left(0, \frac{1}{2}\right)$. Therefore, $f(\psi, \theta)<0$ for $\forall \psi \in\left(0, \frac{1}{3}\right)$ and $\forall \theta \in\left(0, \frac{1}{2}\right)$, which implies $\partial^{2} \pi_{M}^{*}(\theta, \psi) / \partial \theta \partial \psi<0$. Similarly, $\partial^{2} \pi_{N}^{*}(\theta, \psi) / \partial \theta \partial \psi=$ $g(\psi, \theta) /\left((1-\theta)^{2}(3-3 \theta-3 \psi+7 \theta \psi)^{4}\right)$, where $g(\psi, \theta)$ is a polynomial function of $\psi$ and $\theta$ with degree of $10\left(\psi^{4} \theta^{6}\right)$. Again, we can show that $g(\psi, \theta)$ is convex in $\psi$ for $0<\psi<\frac{1}{3}$ and $0<\theta<\frac{1}{2}$. Because $g(0, \theta)<0$ and $g\left(\frac{1}{3}, \theta\right)<0$ for $\forall \theta \in$ $\left(0, \frac{1}{2}\right)$, we can conclude that $g(\psi, \theta)<0$ for $\forall \psi \in\left(0, \frac{1}{3}\right)$ and $\forall \theta \in\left(0, \frac{1}{2}\right)$. Therefore, $\partial^{2} \pi_{N}^{*}(\theta, \psi) / \partial \theta \partial \psi<0$.

Proof of Proposition 1. Define $h(\theta, \psi)=\pi_{M}^{*}(\theta, \psi)+$ $\pi_{N}^{*}(\theta, \psi)$. Because both $\pi_{M}^{*}(\theta, \psi)$ and $\pi_{N}^{*}(\theta, \psi)$ are submodular by Lemma $1, h(\theta, \psi)$ is also submodular. We then define

$$
\begin{aligned}
H\left(\theta, \psi_{1}, \psi_{2}\right) \equiv & {\left[\pi_{M}^{*}\left(\theta, \psi_{2}\right)-\pi_{M}^{*}\left(\theta, \psi_{1}\right)\right] } \\
& -\left[\pi_{N}^{*}\left(\theta, \psi_{1}\right)-\pi_{N}^{*}\left(\theta, \psi_{2}\right)\right] \\
= & h\left(\theta, \psi_{2}\right)-h\left(\theta, \psi_{1}\right) .
\end{aligned}
$$

If $0<\psi_{1}<\psi_{2}<\frac{1}{3}$ or $\frac{1}{3} \leq \psi_{1}<\psi_{2}<1$, then

$$
\begin{aligned}
\frac{\partial H}{\partial \theta}\left(\theta, \psi_{1}, \psi_{2}\right) & =\frac{\partial h}{\partial \theta}\left(\theta, \psi_{2}\right)-\frac{\partial h}{\partial \theta}\left(\theta, \psi_{1}\right) \\
& =\frac{\partial^{2} h}{\partial \psi \partial \theta}(\theta, \bar{\psi})\left(\psi_{2}-\psi_{1}\right),
\end{aligned}
$$

where the second equality is by applying the mean value theorem and $\bar{\psi} \in\left[\psi_{1}, \psi_{2}\right]$. Because $\left(\partial^{2} h / \partial \psi \partial \theta\right)(\theta, \bar{\psi})<0$, $(\partial H / \partial \theta)\left(\theta, \psi_{1}, \psi_{2}\right)<0$.

$$
\begin{aligned}
& \text { If } 0<\psi_{1}<\frac{1}{3} \leq \psi_{2}<1 \text {, then } \\
& \begin{aligned}
\frac{\partial H}{\partial \theta}\left(\theta, \psi_{1}, \psi_{2}\right) & =\frac{\partial h}{\partial \theta}\left(\theta, \psi_{2}\right)-\frac{\partial h}{\partial \theta} h\left(\theta, \psi_{1}\right) \\
& =\int_{\psi_{1}}^{1 / 3} \frac{\partial^{2} h}{\partial \psi \partial \theta}(\theta, \psi) d \psi+\int_{1 / 3}^{\psi_{2}} \frac{\partial^{2} h}{\partial \psi \partial \theta}(\theta, \psi) d \psi .
\end{aligned}
\end{aligned}
$$

Because $\left(\partial^{2} h / \partial \psi \partial \theta\right)(\theta, \psi)<0,(\partial H / \partial \theta)\left(\theta, \psi_{1}, \psi_{2}\right)<0$.

In sum, $H\left(\theta, \psi_{1}, \psi_{2}\right)$ is decreasing in $\theta$. Notice that $H\left(0, \psi_{1}, \psi_{2}\right)=5\left(\psi_{2}-\psi_{1}\right) /\left(9\left(1-\psi_{1}\right)\left(1-\psi_{2}\right)\right)$ (in the case of $\left.0<\psi_{1}<\psi_{2}<\frac{1}{3}\right)$, or $\left(\psi_{2}-\psi_{1}\right) / 4$ (in the case of $\frac{1}{3} \leq \psi_{1}<$ $\left.\psi_{2}<1\right)$, or $\left(20-9\left(1-\psi_{2}\right)\left(3+\psi_{1}\right)\right) /\left(36\left(1-\psi_{2}\right)\right)$ (in the case of $\left.0<\psi_{1}<\frac{1}{3} \leq \psi_{2}<1\right)$. We can verify that $H\left(0, \psi_{1}, \psi_{2}\right)>0$ in all three cases. Similarly, $H\left(\frac{1}{2}, \psi_{1}, \psi_{2}\right)=-\left(\psi_{2}-\psi_{1}\right) / 2<0$ in all three cases. Because $H\left(\theta, \psi_{1}, \psi_{2}\right)$ is continuous in $\theta$, we can conclude that there exists a cutoff $\theta^{*} \in\left(0, \frac{1}{2}\right)$ such that $H\left(\theta^{*}, \psi_{1}, \psi_{2}\right)=0, H\left(\theta, \psi_{1}, \psi_{2}\right)>0$ for $\theta<\theta^{*}$, and $H\left(\theta, \psi_{1}, \psi_{2}\right)<0$ for $\theta>\theta^{*}$. Notice that $\pi_{M}^{*}\left(\theta, \psi_{2}\right)-$ $\pi_{M}^{*}\left(\theta, \psi_{1}\right)>0$ always holds, that is, $b_{M}^{*}>0$. Therefore, we can conclude that $b_{M}^{*}>b_{N}^{*}$ for $\theta<\theta^{*}$, and $b_{M}^{*}<b_{N}^{*}$ for $\theta>\theta^{*}$.

Proof of Corollary 1. According to Table 2, when $\psi<$ $\frac{1}{3}, \partial \pi_{N}^{*}(\theta, \psi) / \partial \psi=([(1+\theta)(1-\theta)+\theta(3 \theta-1) \psi] f(\psi, \theta)) /$ $\left((1-\theta)[3(1-\theta)(1-\psi)+4 \theta \psi]^{3}\right)$, where $f(\psi, \theta)$ is a polynomial function of $\psi$ and $\theta$ with degree of $5\left(\psi^{2} \theta^{3}\right)$. We have $\partial f(\psi, \theta) / \partial \psi=-\theta(1-3 \theta)(3-7 \theta) \psi-(1-\theta)\left(20 \theta^{2}-13 \theta+3\right)$. Within the given parameter region, $\partial f(\psi, \theta) / \partial \psi<0$. Notice that $f\left(\frac{1}{3}, \theta\right)=\frac{2}{9}(3-\theta)\left(12 \theta^{2}-17 \theta+3\right)$. When $\theta<$ $(17-\sqrt{145}) / 24, f\left(\frac{1}{3}, \theta\right)>0$, which implies $f(\psi, \theta)>0$ for $\forall \psi \in\left(0, \frac{1}{3}\right)$. Therefore, $\partial \pi_{N}^{*}(\theta, \psi) / \partial \psi>0$, and thus $\pi_{N}^{*}\left(\theta, \psi_{1}\right)-\pi_{N}^{*}\left(\theta, \psi_{2}\right)<0$ and $b_{N}^{*}=0$.

Proof of Proposition 2. We let $\Delta \hat{\pi}_{i}=\hat{\pi}_{i}^{1}-\hat{\pi}_{i}^{2}, i \in$ $\{M, N\}$. When $\frac{1}{3} \leq \psi<1$, according to Table $3, \Delta \hat{\pi}_{M}-\Delta \hat{\pi}_{N}=$ $\left[(1-2 \theta) /\left(4(3+\psi)^{2}(1-\theta)\right)\right] F(\psi, \theta)$, where

$F(\psi, \theta)=-4\left(\psi^{3}+3 \psi^{2}+5 \psi-1\right) \theta+\left(5 \psi^{3}+17 \psi^{2}+19 \psi+7\right)$.

Because $F(\psi, 0)>0, F\left(\psi, \frac{1}{2}\right)>0$, and $F(\psi, \theta)$ is linear in $\theta$, we can conclude that $F(\psi, \theta)>0$ and hence $\Delta \hat{\pi}_{M}-\Delta \hat{\pi}_{N}>0$ for $\forall \theta \in\left(0, \frac{1}{2}\right)$.

When $0<\psi<\frac{1}{3}$, similarly, $\Delta \hat{\pi}_{M}-\Delta \hat{\pi}_{N}=[\psi(1-2 \theta) /$ $\left.\left((1-\theta)(3+\psi)^{2}(3-3 \psi-3 \theta+7 \theta \psi)^{2}\right)\right] G(\psi, \theta)$, where $G(\psi, \theta)$ is a polynomial function of $\psi$ and $\theta$ with degree of $7\left(\psi^{4} \theta^{3}\right)$. As it can be verified that $G\left(\psi, \frac{1}{2}\right)>0$ and $\partial G(\psi, \theta) / \partial \theta<0$ for $\forall \theta \in\left(0, \frac{1}{2}\right), G(\psi, \theta)>0$ and thus $\Delta \hat{\pi}_{M}-\Delta \hat{\pi}_{N}>0$ for $\forall \theta \in\left(0, \frac{1}{2}\right)$.

We can further check that $\hat{b}_{M}=\left[\Delta \hat{\pi}_{M}\right]^{+}>0$. Therefore, $\hat{b}_{M}>\hat{b}_{N}$ for $\forall \psi \in(0,1)$ and $\forall \theta \in\left(0, \frac{1}{2}\right)$.

Proof of Corollary 2. (i) When $\psi<\frac{1}{3}$, according to Table $3, \hat{\pi}_{N}^{1}-\hat{\pi}_{N}^{2}$ can be simplified as $\psi F(\psi, \theta) /((1-\theta)$. $\left.(3+\psi)^{2}[3(1-\theta)(1-\psi)+4 \theta \psi]^{2}\right)$, where $F(\psi, \theta)$ is a polynomial function of $\psi$ and $\theta$ with degree of $8\left(\theta^{4} \psi^{4}\right)$. We can show that $\left(\partial^{2} F / \partial \theta^{2}\right)(\psi, \theta)$ is monotonic in $\theta$ for $\forall \theta \in$ $\left(0, \frac{1}{2}\right)$, and both $\left(\partial^{2} F / \partial \theta^{2}\right)(\psi, 0)$ and $\left(\partial^{2} F / \partial \theta^{2}\right)\left(\psi, \frac{1}{2}\right)$ are negative. Therefore, $\left(\partial^{2} F / \partial \theta^{2}\right)(\psi, \theta)<0$, and thus $(\partial F / \partial \theta)(\psi, \theta)$ is decreasing in $\theta$. We can further verify that $(\partial F / \partial \theta)\left(\psi, \frac{1}{2}\right)>0$ and thus $(\partial F / \partial \theta)(\psi, \theta)>0$ for $\forall \theta \in\left(0, \frac{1}{2}\right)$. We also notice that $F\left(\frac{1}{3}, 5 \sqrt{6} / 6-2\right)=0$ and $F(\psi, 5 \sqrt{6} / 6-2)$ is decreasing in $\psi$, which leads to $F(\psi, 5 \sqrt{6} / 6-2)>0$ for $\forall \psi \in\left(0, \frac{1}{3}\right)$. Therefore, $F(\psi, \theta)>0$ and $\hat{\pi}_{N}^{1}>\hat{\pi}_{N}^{2}$ for $\forall \theta \in\left(5 \sqrt{6} / 6-2, \frac{1}{2}\right)$ and $\forall \psi \in\left(0, \frac{1}{3}\right)$. 
(ii) When $\psi \geq \frac{1}{3}, \hat{\pi}_{N}^{2}$ is decreasing in $\psi$. If we view $\hat{\pi}_{N}^{1}$ and $\hat{\pi}_{N}^{2}$ as functions of $\psi$, it is sufficient to show $\hat{\pi}_{N}^{1}(\psi)>\hat{\pi}_{N}^{2}\left(\frac{1}{3}\right)$ for $\forall \psi \geq \frac{1}{3}$. Notice that $\hat{\pi}_{N}^{1}(\psi)-\hat{\pi}_{N}^{2}\left(\frac{1}{3}\right)=$ $G(\psi, \theta) /\left(6(1-\theta)(3+\psi)^{2}\right)$, where $G(\psi, \theta)=6(1-\psi)^{2} \theta^{2}+$ $12\left(1-\psi^{2}\right) \theta+\left(5 \psi^{2}+6 \psi-3\right)$. Because $\partial G / \partial \theta>0, \partial G / \partial \psi>0$, and $G\left(\frac{1}{3}, 5 \sqrt{6} / 6-2\right)=0, G(\psi, \theta)>0$ and thus $\hat{\pi}_{N}^{1}(\psi)>$ $\hat{\pi}_{N}^{2}\left(\frac{1}{3}\right)$ for $\forall \psi \in\left[\frac{1}{3}, 1\right)$ and $\forall \theta \in\left(5 \sqrt{6} / 6-2, \frac{1}{2}\right)$.

Proof of Proposition 3. We plug in $p_{M}^{*}(\theta, \psi)$ and $p_{N}^{*}(\theta, \psi)$ from Equation (5) into Equation (10), and want to show $\partial W(\theta, \psi) / \partial \psi<0$.

(i) When $\psi<\frac{1}{3}, \partial W(\theta, \psi) / \partial \psi=F(\psi, \theta) /(2(1-\theta)[3(1-\theta)$. $\left.(1-\psi)+4 \theta \psi]^{3}\right)$, where $F(\psi, \theta)$ is a polynomial function of $\psi$ and $\theta$ with degree of $8\left(\theta^{5} \psi^{3}\right)$. By its concavity $\left(\partial^{2} F(\psi, \theta) / \partial \psi^{2}<0\right)$, we can show that $F(\psi, \theta)<0$ for $\forall \psi \in$ $(0,1)$. Therefore, $\partial W(\theta, \psi) / \partial \psi<0$, for $\forall \psi \in\left(0, \frac{1}{3}\right)$ and $\forall \theta \in$ $\left(0, \frac{1}{2}\right)$.

(ii) When $\psi \geq \frac{1}{3}, \partial W(\theta, \psi) / \partial \psi=\left(12 \theta^{2}-12 \theta+1\right) /(8(1-\theta))$, which is negative when $\theta>\frac{1}{2}-\sqrt{6} / 6$. When $\theta<\frac{1}{2}-\sqrt{6} / 6$, we can check that $M$ outbids $N$ in both cases, and therefore $W^{B}=W^{C}$.

Altogether, according to Propositions 1 and 2, when $\theta>\theta^{*}\left(\psi_{1}, \psi_{2}\right), W^{C}=W\left(\theta, \psi_{1}\right)>W\left(\theta, \psi_{2}\right)=W^{B}$; when $\theta<$ $\theta^{*}\left(\psi_{1}, \psi_{2}\right), W^{C}=W^{B}=W\left(\theta, \psi_{2}\right)$.

Proof of Proposition 4. (i) When $\psi<\frac{1}{3}, D_{M}(\psi)=$ $([(2-\theta)+\theta \psi][(1-\theta)-(1-2 \theta) \psi]) /(3(1-\theta)(1-\psi)+$ $4 \theta \psi)$ and $D_{N}(\psi)=([(1+\theta)(1-\theta)+\theta(3 \theta-1) \psi](1-\psi)) /$ $(3(1-\theta)(1-\psi)+4 \theta \psi)$. By Equation (12), we have $\partial G(\psi) / \partial \psi=\theta F(\psi, \theta) /\left(-3+3 \theta+3 \psi-5 \theta \psi-\theta^{2} \psi+\theta^{2} \psi^{2}\right)^{2}$, where $F(\psi, \theta)$ is a quadratic function of $\psi$ and can be proved positive. (If we write $F(\psi, \theta)$ as $A \psi^{2}+B \psi+C$, we can show $A>0$ and $B^{2}-4 A C<0$.) Therefore, $\partial G(\psi) / \partial \psi>0$, for $\forall \psi \in\left(0, \frac{1}{3}\right)$ and $\forall \theta \in\left(0, \frac{1}{2}\right)$. (ii) When $\psi \geq \frac{1}{3}, D_{M}(\psi)=$ $\left(\frac{1}{2}-\theta\right) \psi+\frac{1}{2}$ and $D_{N}(\psi)=\frac{1}{2}-\psi / 2$. Therefore, $G(\psi)=$ $(1-\theta) \psi /(2(1-\theta \psi))$, which is increasing in $\psi$. Altogether, $\partial G(\psi) / \partial \psi>0$ for $\forall \theta \in\left(0, \frac{1}{2}\right)$ and thus, according to Propositions 1 and $2, G^{C}=G\left(\psi_{1}\right)<G\left(\psi_{2}\right)=G^{B}$ when $\theta>\theta^{*}\left(\psi_{1}, \psi_{2}\right)$, and $G^{C}=G^{B}=G\left(\psi_{2}\right)$ when $\theta<\theta^{*}\left(\psi_{1}, \psi_{2}\right)$.

Proof of Proposition 5. Recall that $\hat{b}_{N}=\left[\hat{\pi}_{N}^{1}-\hat{\pi}_{N}^{2}\right]^{+}$, $b_{N}^{*}=\left[\pi_{N}^{*}\left(\theta, \psi_{1}\right)-\pi_{N}^{*}\left(\theta, \psi_{2}\right)\right]^{+}$, and $\hat{\pi}_{N}^{2}=\pi_{N}^{*}\left(\theta, \psi_{2}\right)$. By Table $3, \hat{\pi}_{N}^{1}$ is increasing in $\psi$ (by simply checking the first-order derivative) and thus $\hat{\pi}_{N}^{1}\left(\psi_{2}\right)>\hat{\pi}_{N}^{1}\left(\psi_{1}\right)$ for all $0<$ $\psi_{1}<\psi_{2}<1$. From Corollary 2, $\hat{\pi}_{N}^{1}(\psi)>\hat{\pi}_{N}^{2}(\psi)$ for any $\psi \in$ $(0,1)$ when $\theta \in\left(5 \sqrt{6} / 6-2, \frac{1}{2}\right)$. Therefore, $\hat{\pi}_{N}^{1}\left(\psi_{2}\right)>\hat{\pi}_{N}^{2}\left(\psi_{1}\right)$. As $\hat{\pi}_{N}^{2}\left(\psi_{1}\right)=\pi_{N}^{*}\left(\theta, \psi_{1}\right)$ (because of the same information structure), $\hat{\pi}_{N}^{1}\left(\psi_{2}\right)-\hat{\pi}_{N}^{2}>\pi_{N}^{*}\left(\theta, \psi_{1}\right)-\pi_{N}^{*}\left(\theta, \psi_{2}\right)$. When $\theta \in$ $\left(5 \sqrt{6} / 6-2, \frac{1}{2}\right)$, because $\hat{b}_{N}>0$ by Corollary $2, I R^{B}=\hat{b}_{N}>$ $b_{N}^{*} \geq \min \left\{b_{M}^{*}, b_{N}^{*}\right\}=I R^{C}$ for all $0<\psi_{1}<\psi_{2}<1$.

Proof of Proposition 6. We plug in $p_{M}^{*}(\theta, \psi)$ and $p_{N}^{*}(\theta, \psi)$ from Equation (5) into Equation (13), and want to show $\partial \operatorname{CS}(\theta, \psi) / \partial \psi<0$. (i) When $\psi<\frac{1}{3}, \partial \operatorname{CS}(\theta, \psi) / \partial \psi=$ $(1-2 \theta) F(\psi, \theta) /\left(2(1-\theta)[3(1-\theta)(1-\psi)+4 \theta \psi]^{3}\right)$, where $F(\psi, \theta)$ is a polynomial function of $\psi$ and $\theta$ with degree of $7\left(\theta^{4} \psi^{3}\right)$. As we can show, for $\forall \psi \in\left(0, \frac{1}{3}\right), \partial F(\psi, \theta) / \partial \psi>0$ when $\theta<\frac{3}{7}$, and $\partial^{2} F(\psi, \theta) / \partial \psi^{2}>0$ when $\theta>\frac{3}{7}$. In other words, $F(\psi, \theta)$ is either increasing or convex in $\psi$. Notice that $F(0, \theta)=(1-\theta)^{2}\left(-33-8 \theta+22 \theta^{2}\right)<0$ and $F\left(\frac{1}{3}, \theta\right)=$ $-(2 / 27)(3-\theta)^{2}\left(33-37 \theta+10 \theta^{2}\right)<0$ for $\forall \theta \in\left(0, \frac{1}{2}\right)$, we can conclude that $F(\psi, \theta)<0$ and hence $C S(\psi, \theta)$ is decreasing in $\psi$ for $\forall \theta \in\left(0, \frac{1}{2}\right), \forall \psi \in\left(0, \frac{1}{3}\right)$. (ii) When $\psi \geq \frac{1}{3}$, we have $C S(\theta, \psi)=((1-2 \theta)(1+2 \theta) /(8(1-\theta)))(1-\psi)$, which is decreasing in $\psi$. Altogether, according to Propositions 1 and 2, $C^{C}=C S\left(\theta, \psi_{1}\right)>C S\left(\theta, \psi_{2}\right)=\operatorname{CS}^{B}$ when $\theta>\theta^{*}\left(\psi_{1}, \psi_{2}\right)$, and $C S^{C}=C S^{B}=C S\left(\theta, \psi_{2}\right)$ when $\theta<\theta^{*}\left(\psi_{1}, \psi_{2}\right)$.

Proof of Proposition 7. We first derive the secondstage equilibrium prices. When $M$ wins the first sponsored slot, for $\psi_{2}<1 /(3(1-\lambda))$,

$$
\left\{\begin{array}{l}
\tilde{p}_{M}^{1}=\frac{(1-\lambda) \theta \psi_{2}+2-\theta}{4(1-\lambda) \psi_{2} \theta+3(1-\lambda)\left(1-\psi_{2}\right)(1-\theta)+3 \lambda(1-\theta)} \\
\tilde{p}_{N}^{2}=\frac{(1-\lambda) \psi_{2} \theta(3 \theta-1)+1-\theta^{2}}{(1-\theta)\left[4(1-\lambda) \psi_{2} \theta+3(1-\lambda)\left(1-\psi_{2}\right)(1-\theta)+3 \lambda(1-\theta)\right]} .
\end{array}\right.
$$

When $N$ wins the first sponsored slot,

$$
\left\{\begin{array}{l}
\tilde{p}_{M}^{2}=\frac{\lambda \psi_{2}(1-\theta)+2-\theta}{(1-\theta)\left(\lambda \psi_{2}+3\right)} \\
\tilde{p}_{N}^{1}=\frac{\lambda \psi_{2}(1-\theta)+1+\theta}{(1-\theta)\left(\lambda \psi_{2}+3\right)} .
\end{array}\right.
$$

(i) Substituting equilibrium prices in Equation (21) into the welfare function in Equation (16), we can derive $\partial \tilde{W}\left(\theta, \psi_{2}, \lambda\right) /\left.\partial \lambda\right|_{\lambda=0}=\psi_{2} H\left(\psi_{2}, \theta\right) /\left(2(1-\theta)\left[4 \psi_{2} \theta+3\left(1-\psi_{2}\right)\right.\right.$. $\left.(1-\theta)]^{3}\right)$, where $H\left(\psi_{2}, \theta\right)$ is a polynomial function of $\psi_{2}$ and $\theta$ with degree of $8\left(\theta^{5} \psi_{2}^{3}\right)$. Following similar arguments as in (iv), we can show that $H\left(\psi_{2}, \theta\right)$ is decreasing in $\psi_{2}$ for $\forall \psi_{2} \in(0,1)$ and $\forall \theta \in\left(0, \theta_{0}\right)$. Furthermore, $H\left(\frac{1}{3}, \theta\right)=(2 / 27)(3-\theta)^{2}\left[\theta\left(12\left(\theta-\frac{3}{2}\right)^{2}-8\right)+3\right]>0$ for $0<\theta<$ $\frac{1}{2}$. Therefore, $H\left(\psi_{2}, \theta\right)>0$, and thus $\partial \tilde{W}\left(\theta, \psi_{2}, \lambda\right) /\left.\partial \lambda\right|_{\lambda=0}>0$ for $\forall \psi_{2} \in(0,1 /(3(1-\lambda)))$ and $\forall \theta \in\left(0, \theta_{0}\right)$.

(ii) The sales Gini coefficient in equilibrium is

$$
\tilde{G}\left(\theta, \psi_{2}, \lambda\right)=\frac{1}{2}-\frac{D_{N}^{2}\left(\tilde{p}_{N}^{2}, \tilde{p}_{M}^{1}\right)}{D_{M}^{1}\left(\tilde{p}_{M}^{1}, \tilde{p}_{N}^{2}\right)+D_{N}^{2}\left(\tilde{p}_{N}^{2}, \tilde{p}_{M}^{1}\right)} .
$$

Substituting equilibrium prices in Equation (21) into this expression, we can derive $\partial \tilde{G}\left(\theta, \psi_{2}, \lambda\right) /\left.\partial \lambda\right|_{\lambda=0}=\left(\theta \psi_{2}\left(A \psi_{2}^{2}+\right.\right.$ $\left.\left.B \psi_{2}+C\right)\right) /\left(\theta^{2} \psi_{2}^{2}-\theta^{2} \psi_{2}-5 \theta \psi_{2}+3 \theta+3 \psi_{2}-3\right)^{2}$, where $A=\theta^{3}-15 \theta^{2}+13 \theta-3, B=-2 \theta^{3}+18 \theta^{2}-22 \theta+6$, and $C=\theta^{3}-7 \theta^{2}+11 \theta-5$. We can verify that $B^{2}-4 A C<0$ and $A<0$, and therefore $\partial \tilde{G}\left(\theta, \psi_{2}, \lambda\right) /\left.\partial \lambda\right|_{\lambda=0}<0$ for $\forall \psi_{2} \in$ $(0,1 /(3(1-\lambda)))$ and $\forall \theta \in\left(0, \theta_{0}\right)$.

(iii) The equilibrium consumer surplus can be written as

$$
\begin{aligned}
\widetilde{C S}\left(\theta, \psi_{2}, \lambda\right)= & (1-\lambda) \psi_{2}\left[(1-\theta)\left(1-\tilde{p}_{M}^{1}\right)+\theta \int_{\tilde{p}_{M}^{1}}^{1}\left(x-\tilde{p}_{M}^{1}\right) d x\right] \\
& +\left[1-(1-\lambda) \psi_{2}\right](1-\theta)\left(1-\tilde{p}_{M}^{1}+\tilde{p}_{N}^{2}\right)\left(1-\tilde{p}_{M}^{1}\right) \\
& +\left[1-(1-\lambda) \psi_{2}\right]\left[\theta\left(1-\tilde{p}_{N}^{2}\right)\right. \\
& \left.+(1-\theta) \int_{1-\tilde{p}_{M}^{1}+\tilde{p}_{N}^{2}}^{1}\left(x-\tilde{p}_{N}^{2}\right) d x\right] .
\end{aligned}
$$

Substituting equilibrium prices in Equation (21) into this expression, we can derive $\partial \widetilde{C S}\left(\theta, \psi_{2}, \lambda\right) /\left.\partial \lambda\right|_{\lambda=0}=((1-2 \theta)$. $\left.\psi_{2} G\left(\psi_{2}, \theta\right)\right) /\left(2(1-\theta)\left[4 \psi_{2} \theta+3\left(1-\psi_{2}\right)(1-\theta)\right]^{3}\right)$, where $G\left(\psi_{2}, \theta\right)$ is a polynomial function of $\psi_{2}$ and $\theta$ with degree of seven $\left(\theta^{4} \psi_{2}^{3}\right)$. We can show that for $\forall \psi_{2} \in(0$, $1 /(3(1-\lambda))), G\left(\psi_{2}, \theta\right)$ is decreasing in $\psi_{2}$ when $\theta<\frac{3}{7}$, and $G\left(\psi_{2}, \theta\right)$ is concave in $\psi_{2}$ when $\theta>\frac{3}{7}$. Notice that $G(0, \theta)=$ $(1-\theta)^{2}\left(33+8 \theta-22 \theta^{2}\right)>0$ and $G\left(\frac{1}{3}, \theta\right)=(2 / 27)(3-\theta)^{2}$. 
$\left(33-37 \theta+10 \theta^{2}\right)>0$, we can conclude that $G\left(\psi_{2}, \theta\right)>0$ and hence $\partial \widetilde{C S}\left(\theta, \psi_{2}, \lambda\right) /\left.\partial \lambda\right|_{\lambda=0}>0$ for $\forall \psi_{2} \in(0,1 /(3(1-\lambda)))$ and $\forall \theta \in\left(0, \theta_{0}\right)$.

(iv) We can calculate $N^{\prime}$ s equilibrium profit in both cases: $\tilde{\pi}_{N}^{1}=\tilde{p}_{N}^{1} D_{N}^{1}\left(\tilde{p}_{N}^{1}, \tilde{p}_{M}^{2}\right)$ and $\tilde{\pi}_{N}^{2}=\tilde{p}_{N}^{2} D_{N}^{2}\left(\tilde{p}_{N}^{2}, \tilde{p}_{M}^{1}\right)$ according to Equations (15) and (14). We then have $\Delta \tilde{\pi}_{N}\left(\theta, \psi_{2}, \lambda\right)=$ $\tilde{\pi}_{N}^{1}-\tilde{\pi}_{N}^{2}$, and $\partial \Delta \tilde{\pi}_{N}\left(\theta, \psi_{2}, \lambda\right) /\left.\partial \lambda\right|_{\lambda=0}=\psi_{2} F\left(\psi_{2}, \theta\right) /(27(1-\theta)$. $\left.\left[4 \psi_{2} \theta+3\left(1-\psi_{2}\right)(1-\theta)\right]\right)$, where $F\left(\psi_{2}, \theta\right)$ is a polynomial function of $\psi_{2}$ and $\theta$ with degree of $8\left(\theta^{5} \psi_{2}^{3}\right)$. We can show that $\partial^{2} F\left(\psi_{2}, \theta\right) / \partial \psi_{2}^{2}=f(\theta)\left[4 \psi_{2} \theta+3\left(1-\psi_{2}\right)(1-\theta)\right]$, where $f(\theta)$ is an expression that only contains $\theta$. For a given $\theta, \partial^{2} F\left(\psi_{2}, \theta\right) / \partial \psi_{2}^{2}$ does not change sign for $\forall \psi_{2} \in(0,1)$; that is, $\left(\partial F / \partial \psi_{2}\right)\left(\psi_{2}, \theta\right)$ is monotonic in $\psi_{2}$. We can check that $\left(\partial F / \partial \psi_{2}\right)(0, \theta)<0$ and $\left(\partial F / \partial \psi_{2}\right)(1, \theta)<0, \forall \theta \in\left(0, \frac{1}{2}\right)$. Therefore, $\left(\partial F / \partial \psi_{2}\right)\left(\psi_{2}, \theta\right)<0$ for $\forall \psi_{2} \in(0,1)$ and $\forall \theta \in$ $\left(0, \frac{1}{2}\right)$. Because we focus on the case where $\psi_{2}<1 /(3(1-\lambda))$ $\left(=\frac{1}{3}\right.$ at $\left.\lambda=0\right)$ and we notice that $F\left(\frac{1}{3}, \theta\right)=(2 / 27)(3-\theta)^{2}$. $\left(32 \theta^{3}+244 \theta^{2}-523 \theta+129\right)$ crosses zero from above once when changing $\theta$ from 0 to $1, F\left(\frac{1}{3}, \theta\right)>0$ when $\theta \in\left(0, \theta_{0}\right)$. By monotonicity of $F, F\left(\psi_{2}, \theta\right)>0$ and thus $\partial \Delta \tilde{\pi}_{N}\left(\theta, \psi_{2}, \lambda\right) /\left.\partial \lambda\right|_{\lambda=0}>0$, for $\forall \psi_{2} \in(0,1 /(3(1-\lambda)))$ and $\forall \theta \in\left(0, \theta_{0}\right)$. We can further check that $\theta_{0}<\theta^{*}\left(0, \psi_{2}\right)$ (as defined by Equation (7)) for the given parameter region, which validates the presumed bidding outcome (i.e., $b_{N}<b_{M}$ ).

\section{References}

Agarwal A, Hosanager K, Smith MD (2012) Do organic results help or hurt sponsored search performance? Working paper, http://ssrn.com/abstract=1989590.

Athey S, Ellison G (2010) Position auctions with consumer search. Quart. J. Econom. 126(3):1213-1270.

Bagwell K (2007) The Economic Analysis of Advertising, Handbook of Industrial Organization, Vol. 3, Chap. 28 (North-Holland, Amsterdam), 1701-1844.

Butters GR (1977) Equilibrium distributions of sales and advertising prices. Rev. Econom. Stud. 44(3):465-491.

Casadesus-Masanell R, Zhu F (2010) Strategies to fight ad-sponsored rivals. Management Sci. 56(9):1484-1499.

Casadesus-Masanell R, Zhu F (2012) Business model innovation and competitive imitation: The case of sponsor-based business models. Strategic Management J. Forthcoming.
Dellarocas C. (2011) Double marginalization in performancebased advertising: Implications and solutions. Management Sci. 58(6):1178-1195

Edelman B, Ostrovsky M, Schwarz M (2007) Internet advertising and the generalized second-price auction: Selling billions of dollars worth of keywords. Amer. Econom. Rev. 97(1): 242-259.

Grossman GM, Shapiro C (1984) Informative advertising with differentiated products. Rev. Econom. Stud. 51(1):63-81.

Hotchkiss G, Alston S, Edwards G (2005) Eye tracking study. Research white paper, Enquiro Search Solutions Inc. http:// www.enquireresearch.com/images/eyetracking2-sample.pdf.

IAB, PwC (2012) IAB Internet advertising revenue report 2011 full-year results. Market research report, Interactive Advertising Bureau (IAB) and PricewaterhouseCoopers (PwC). http://www.iab.net/media/file/IAB_Internet_Advertising _Revenue_Report_FY_2011.pdf.

Katona Z, Sarvary M (2008) Network formation and the structure of the commercial World Wide Web. Marketing Sci. 27(5): 764-778.

Katona Z, Sarvary M (2010) The race for sponsored links: Bidding patterns for search advertising. Marketing Sci. 29(2):199-215.

Liu D, Chen J, Whinston AB (2010) Ex-ante information and design of keyword auctions. Inform. Systems Res. 21(1):133-153.

Lutz RJ (1985) Affective and cognitive antecedents of attitude towards the ad: A conceptual framework. Psychological Processes and Advertising Effects: Theory, Research, and Applications (Lawrence Erlbaum Associates, Hillsdate, NJ).

Stahl DO, II (1994) Oligopolistic pricing and advertising. J. Econom. Theory 64(1):162-177.

Stegeman M (1991) Advertising in competitive markets. Amer. Econom. Rev. 81(1):210-223.

Weber TA, Zheng Z (2007) A model of search intermediaries and paid referrals. Inform. Systems Res. 18(4):414-436.

White A (2009) Search engines: Left side quality versus right side profits. Working paper, http://ssrn.com/abstract=1694869.

Xu L, Chen J, Whinston A (2011a) Oligopolistic pricing with online search. J. Management Inform. Systems 27(3):111-141.

Xu L, Chen J, Whinston A (2011b) Price competition and endogenous valuation in search advertising. J. Marketing Res. 48(3): 566-586.

Yang S, Ghose A (2010) Analyzing the relationship between organic and sponsored search advertising: Positive, negative, or zero interdependence? Marketing Sci. 29(4):602-623.

Zhang X(Michael), Feng J (2011) Cyclical bid adjustments in searchengine advertising. Management Sci. 57(9):1703-1719. 